PHYSICAL REVIEW D 73, 026003 (2006)

\title{
Stringy effects during inflation and reheating
}

\author{
Andrew R. Frey* \\ California Institute of Technology, 452-48, Pasadena, California 91125, USA
}

Anupam Mazumdar ${ }^{\dagger}$

NORDITA, Blegdamsvej -17, DK-2100, Copenhagen, Denmark

Robert C. Myers

Perimeter Institute for Theoretical Physics, Waterloo, Ontario N2L 2Y5, Canada

and Department of Physics, University of Waterloo, Waterloo, Ontario N2L 3G1, Canada

(Received 28 September 2005; published 23 January 2006)

\begin{abstract}
We consider inflationary cosmology in the context of string compactifications with multiple throats. In scenarios where the warping differs significantly between throats, string and Kaluza-Klein physics can generate potentially observable corrections to the cosmology of inflation and reheating. First we demonstrate that a very low string scale in the ground state compactification is incompatible with a high Hubble scale during inflation, and we propose that the compactification geometry is altered during inflation. In this configuration, the lowest scale is just above the Hubble scale, which is compatible with the effective field theory but still leads to potentially observable cosmic microwave background corrections. Also in the appropriate region of parameter space, we find that reheating leads to a phase of long open strings in the standard model sector (before the usual radiation-dominated phase). We sketch the cosmology of the long string phase and we discuss possible observational consequences.
\end{abstract}

DOI: 10.1103/PhysRevD.73.026003

PACS numbers: $11.25 .-\mathrm{w}, 98.80 . \mathrm{Cq}$

\section{INTRODUCTION: SEEING STRINGS IN COSMOLOGY}

With the great success of inflationary cosmology in explaining the spectrum of cosmic microwave background (CMB) fluctuations [1-4], as well as recent progress in understanding inflation in string theory - see, for example, [5-21] — the time is ripe to ask what signals string theory might have in cosmology. Historically speaking, there has not been much cause for optimism. If strings (or other high-energy physics, such as quantum gravity) modify the four-dimensional effective field theory (EFT) above an energy scale $M$, corrections to the usual Gaussian, scale-invariant CMB spectrum enter at order $(H / M)^{n}$, with $n=2$ or 1 . The value of $n$ has been a matter of some debate in the literature. Standard EFT arguments give $n=2[22]$ unless boundary terms are added [2330]. The latter may yield $n \sim 1$ and seem to reproduce the effects of an unconventional initial state or nonadiabatic evolution [31-47]. However, the main issue remains the conspicuously small value of $H / M$. Experimental data appear to constrain $H / M_{P} \lesssim 10^{-4}$. Even if the scale of new physics is decoupled from the Planck scale, an optimistic bound seems to be $H / M \lesssim 10^{-2}$ for realistic string compactifications [22], even including $\mathrm{TeV}$ gravity scenarios. Except in special cases, for example [48,49], string or higher-dimensional physics seems unobservable. We will argue, however, that a wide class of warped string com-

\footnotetext{
*Electronic address: frey@ theory.caltech.edu

${ }^{\dagger}$ Electronic address: anupamm@nordita.dk

‡Electronic address: rmyers@perimeterinstitute.ca
}

pactifications naturally yields $H / M \sim 1$ during inflation, so that stringy corrections to the EFT are potentially observable. In addition, in the same models, thermal strings may dominate the universe during reheating, leading to other potential cosmological consequences.

In brief, our general argument runs as follows; the key ingredient is warping in compactifications with more than one throat. As explained in [50], warping of the spacetime dimensions generates potentially large hierarchies of scales, possibly creating sectors in which the string and Kaluza-Klein (KK) scales are low. Significant warping can occur in string compactifications, including those described in [51,52], and we will consider models in which the standard model (SM) is confined to branes in a highly warped region (the SM throat). Since these compactifications can also have all moduli frozen, they are suitable both for low-energy phenomenology and inflation [11]. However, we imagine that the inflationary potential is generated in some other region of the compactification (the inflationary throat) than the SM throat. There is a tension between the (usual) high scale of inflation and the low scale of fundamental physics in the SM throat, however, since the 4D curvature $R \sim H^{2}$ can be much larger than the SM sector string scale. It seems that the low-energy description of the SM throat must break down during inflation. We will give this general argument in Sec. II, first reviewing the specific compactifications and inflationary models that we will consider as concrete examples.

The naive idea of inflation occurring on a fixed compactification manifold, however, is in conflict with known 
physics from the 4D EFT. In Sec. III, we will describe a consistent resolution of the tension between Hubble and SM scales. Because of cross couplings and quantum fluctuations, inflation generates Hubble-scale masses for all fields in the EFT [53-55]. From the 10D point of view, the SM throat cannot support such massive modes unless the minimum SM throat warp factor is roughly $H / M_{P}$, so we argue that the compactification does not lie in its ground state during inflation. Specifically, the SM throat is "shorter" than in the post-inflationary ground state, and the SM sector string mass scale is somewhat greater than the Hubble scale of inflation. We end up with a consistent picture; with a large enough SM string scale, the EFT is a reasonable description of the physics after all. As a bonus, the string and KK scales are naturally near the Hubble scale, so high-energy physics will more easily alter the CMB. ${ }^{1}$

After inflation, the question becomes how to reheat the SM degrees of freedom and generate the usual hot big bang cosmology. In particular, in the present scenario, the SM sector is physically separated from the inflationary sector in the compactification geometry. Concentrating on a specific inflationary model, we adopt the argument of [56] that KK modes transmit energy between the two sectors and give a more detailed estimate of the reheating rate in Sec. IV. ${ }^{2}$ We also note that the SM throat will relax to its ground state during reheating, and, in fact, this relaxation will itself reheat the SM sector as well. In the end, we find that, in a large region of parameter space, either (or both) of these reheating processes can yield a reheat temperature larger than the SM throat string scale. In that case, without deriving a string theoretic reheating mechanism, we argue that reheating should lead to a phase of long open strings on the SM branes at the Hagedorn temperature. Some additional comments on reheating, including sketches of reheating in other inflationary models, are relegated to Appendix C.

Reaching the logical conclusion, we discuss the cosmological evolution of open string matter on the SM branes in Sec. V. We first review the thermal distribution of open strings from [59-61] and argue that, unlike closed strings, open strings can easily maintain thermal equilibrium. More details of string thermodynamics are given in Appendix D. We then review the open string decays that can reheat SM radiation [62]; with decay rates in hand, we can then discuss the reheating process from thermal strings to SM radiation. We find that long open strings redshift like matter at high densities, and the open string decays reheat the radiation to a temperature of about the SM string scale. Open strings out of thermal equilibrium also could play a

\footnotetext{
${ }^{1}$ In Appendix B, we discuss alternative possibilities for the SM throat geometry during inflation and give reasons why they do not capture the appropriate physics.

${ }^{2}$ While this work was being completed, a related discussion appeared in $[57,58]$.
}

role in cosmology, and we make some qualitative comments about their evolution.

Finally, we conclude by discussing some possible signals of the low SM string scale, from modifications to the inflationary CMB spectrum to relics of a possible open string phase. Exploring these potential signals is an important direction for future research, especially given the sensitivity of upcoming CMB experiments.

We give a detailed description of our conventions in Appendix A for the interested reader.

\section{THE NAIVE SETTING FOR INFLATION}

To start, we will give a brief review of inflationary models in flux compactifications in a naive form. That is, we will describe the vacuum state of the compactification and then add an inflationary mechanism as a perturbation of this vacuum. Following our review, we will argue that this sort of naive addition of inflation to vacuum compactifications cannot be true in scenarios with multiple throats where there is a significant disparity in the warping of different throats. Specifically, we will demonstrate that Kaluza-Klein and $\alpha^{\prime}$ corrections would necessarily modify the geometry.

\section{A. Vacuum compactification geometry}

Here we will give a brief review of the compactifications we will discuss. For specificity, we will focus on the best understood string compactifications, but our basic conclusions should be rather generic. Therefore, we start by reviewing common features of warped compactifications, which will be the most important for us. Throughout this paper, we will refer to the post-inflationary compactification as the "vacuum" or "ground state" geometry. However, the reader should be aware that, in currently understood models of moduli fixing, these compactifications with a small positive cosmological constant are only metastable and may eventually decompactify to $10 \mathrm{D}$ or suffer other decay modes (though with lifetimes much longer than the age of the universe) $[52,63]$.

Consider, then, a 10D braneworld compactification in which the hierarchy between the Planck and weak scales is provided by a warp factor, as in [50]. We take the metric to be

$$
\begin{aligned}
d s^{2} & =e^{2 A} \eta_{\mu \nu} d x^{\mu} d x^{\nu}+g_{m n}^{6} d x^{m} d x^{n}, \\
& =e^{2 A} \eta_{\mu \nu} d x^{\mu} d x^{\nu}+e^{-2 A} \hat{g}_{m n}^{6} d x^{m} d x^{n}, \quad A \equiv A\left(x^{m}\right) .
\end{aligned}
$$

Here, the internal metric is that of a warped Calabi-Yau manifold - that is $\hat{g}^{6}$, appearing in the second line, is Calabi-Yau [51]. The warp factor provides a hierarchy of scales [50]; at the SM brane(s), $e^{A} \approx M_{\mathrm{SM}} / M_{P}$ implies that $4 \mathrm{D}$ SM observers have a fundamental particle physics scale of $M_{\mathrm{SM}}$ (we use conventions in which $A \sim 0$ away 
from special points of the compactification). ${ }^{3}$ For warping to give the full hierarchy, $M_{\mathrm{SM}} \sim 1 \mathrm{TeV}$. For an intermediate scale hierarchy (with the rest provided, for example, by supersymmetry), simply choose $M_{\mathrm{SM}}$ larger than the weak scale. As will become apparent, the precise value of $M_{\mathrm{SM}}$ will not be important for the following discussion. In a 10D picture, this means that the SM brane is located at the bottom of a throat in the compactification (see, for example, [51] for a string construction). Following the Kaluza-Klein zero-mode for the graviton, the minimal prescription for cosmology is to replace $\eta_{\mu \nu}$ by a cosmological metric $g_{\mu \nu}^{4}$.

Let us examine the hierarchy provided by the warp factor in more detail. Since the warp factor rescales the time coordinate, any localized mode has its mass rescaled by the warp factor, also, as was originally emphasized for Higgs fields in [50]. Direct calculations bear out this expectation in a number of regimes; for example, it is well known that Kaluza-Klein gravitons localized in the SM throat have mass given by the 10D curvature scale times the warp factor, $m \approx e^{A_{\mathrm{SM}}} / R$ [64], where the throat has anti-de Sitter (AdS) geometry and $R$ is the AdS radius. This relation holds even when compactifications have large unwarped regions [65]. Additionally, the tension of long, semiclassical strings (extended in the external directions) is rescaled to $\mu=e^{2 A_{\mathrm{SM}}} / 2 \pi \alpha^{\prime}$, as we can see directly. Taking a static gauge in which the world-sheet spatial coordinate is the target space coordinate length,

$$
\begin{aligned}
S= & -\frac{1}{2 \pi \alpha^{\prime}} \int d^{2} \sigma \sqrt{-\overline{\operatorname{det} g}}, \\
= & -\frac{1}{2 \pi \alpha^{\prime}} \\
& \times \int d^{2} \sigma e^{2 A} \sqrt{1+\left(\partial X^{\mu}\right)^{2}+e^{-2 A}\left(\partial X^{m}\right)^{2}+\cdots}
\end{aligned}
$$

Here $\left(\partial X^{\mu}\right)^{2}$ and $\left(\partial X^{m}\right)^{2}$ schematically represent the fluctuations in the noncompact and compact directions, respectively. Further $\cdots$ represent additional mass terms due to curvature, warping, and possibly other background fields. Hence, we see that strings oscillating purely in 4D have a rescaled tension. Interpolating between KK gravitons and semiclassical strings, we expect that the throat produces a (4D) sector of strings for which all the masses are rescaled by the warp factor. In fact, a perturbative quantization of the string has been done in a slightly different warped background in [66], giving the same result. This fact should lead to similar phenomenological considerations as [67]. For notational clarity, we define the effective string length at the SM brane (located at the tip or bottom of the SM throat, the region of smallest warp

\footnotetext{
${ }^{3}$ See Appendix A for our conventions for the Planck scale, string scale, etcetera.
}

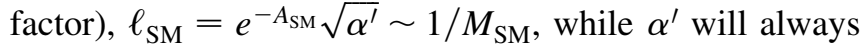
denote the $10 \mathrm{D}$ value.

To be concrete, we will focus on compactifications of the type described by $[51,68,69]$ (see [70] for a review and more references), although our comments should apply qualitatively to any warped string compactification. An important feature of these compactifications is that supergravity 3-form flux generates a potential for many of the compactification moduli, fixing their expectation values. The remaining moduli, including the size modulus $u,{ }^{4}$ can be stabilized by nonperturbative superpotentials generated by D-brane instantons or gaugino condensation $[52,71]$ or potentially by $\alpha^{\prime}$ corrections to the supergravity $[72,73] .^{5}$ Since the vacuum expectation values (VEVs) of the stabilized moduli are model dependent, we can imagine tuning their values to determine what regions of parameter space produce specific physics, and we will typically leave the moduli unspecified. However, we will sometimes give numerical estimates, in which case we will assume that the string coupling $g_{s} \sim 1 / 10$ and the volume $e^{4 u} / g_{s} \sim$ $1-10^{3}$, which includes the example of [52]- the combination $e^{4 u} / g_{s}$ actually corresponds to the real part of the Kähler modulus in the 4D EFT [51].

Recall from (1) that the internal metric is a warped Calabi-Yau manifold, i.e., $g_{m n}^{6}=e^{-2 A} \hat{g}_{m n}^{6}$ with $\hat{g}^{6}$ Calabi-Yau. The warp factor is negligible, $A \sim 0$, away from singularities of the Calabi-Yau, and the geometry develops (locally AdS) throats near the singularities. This warp factor is sourced by the 3 -form fluxes, so the final geometry will depend on the flux quantum numbers $n_{f}$ (see footnote ${ }^{4}$ ). The most studied form of the throats have roughly the geometry of $[75,76]$ and are topologically deformed conifolds. The amount of deformation is controlled by one of the moduli of the Calabi-Yau, and the flux stabilizes the modulus. (In an abuse of language, we will continue to call this scalar a modulus, even though it has a potential, because it will be lighter than other scales we consider.) From the current understanding of the dimensional reduction [74,77-79], the flux-induced mass of the modulus should be $m \sim g_{s} n_{f} / \ell_{\mathrm{SM}}\left(e^{-3 u}+\cdots\right)$, where $n_{f}$ is the number of flux quanta in the throat (defined more precisely in Appendix A) and $\cdots$ represent possible corrections due to derivatives of the warp factor. Most likely, the modulus mass should be between this scale and the KK mass scale, $g_{s} n_{f} e^{-3 u} / \ell_{\mathrm{SM}} \lesssim m \lesssim e^{-A_{\mathrm{SM}}} / R$, simply because the derivatives of the warp factor set the scale $R$. Other throat geometries (based on other possible singularities) have recently been studied in [80-83]. In many cases, the region of the small warp factor $(A \ll 0)$ has been argued to be similar to the deformed conifold. In

\footnotetext{
${ }^{4}$ Again, see definitions in Appendix A.

${ }^{5}$ These nonperturbative and $\alpha^{\prime}$ corrections will modify the geometry we describe here, but those effects are subleading. The recent work of [74] provides the first step towards incorporating these corrections.
} 
particular, in those cases, there are deformation moduli which are also stabilized by supergravity flux. Although there may be other, more exotic throat geometries possible (e.g., a geometric resolution seems to be lacking for cascades ending with dynamical supersymmetry breaking [84-86]), our discussion will apply qualitatively for a large class of them.

There are a number of possibilities for the SM branes. The simplest (though not realistic) choice is a stack of D3 or $\overline{\mathrm{D} 3}$ branes. The antibrane positions are stabilized at the bottom of the SM throat by the warp factor $[52,87]$, and D3-brane positions can be stabilized by nonperturbative physics [11]. Additionally, if there is an orbifold fixed point at the center of the throat, the SM branes can be pinned to the fixed point. More realistic gauge theories can arise in that way [80]. Another alternative is to consider $\mathrm{D} 3 / \overline{\mathrm{D} 3}$ branes at the bottom of the throat intersecting with D7 branes extending through the throats. Much is known about building realistic models from brane intersections; for example, see [88-90] for reviews. In that case, the SM modes are the D3-brane open strings as well as open strings stretching between the D3 branes and D7 branes. The key point for us is that the SM modes will all be localized on branes at the bottom of the SM throat.

Once again, let us emphasize that, although we are focusing on a particular type of compactification, we expect our results to generalize easily.

\section{B. String implementations of inflation}

We will now review several approaches to inflation within the context of the compactification models discussed above. We should note that all of these inflationary mechanisms are typically treated as small perturbations of the compactification geometry in the literature. In this paper, we will mostly discuss the approach known as brane inflation, but we will also mention two other inflationary mechanisms very briefly.

The most popular approach to embedding inflation in string theory is brane inflation $[5,7,8,10,91]$. In this type of model, the inflationary potential is provided by the Coulomb attraction between spacetime-filling branes and antibranes. For brane inflation to avoid destabilizing the compactification, the moduli must be stabilized at a sufficiently high mass scale (and in a deep enough potential well); Ref. [11] showed that the compactifications described in the previous section can provide a suitable framework for brane inflation. ${ }^{6}$ In addition, the requisite $\overline{\mathrm{D} 3}$ branes are fixed at the bottom of a throat by the warp factor, and the length of the throat allows the interbrane potential to be flat enough to support an accelerating cosmological expansion [11]. One difficulty of brane inflation scenarios as described by [11] is a supergravity $\eta$ problem; in the most basic setting for brane inflation, it

${ }^{6}$ For recent refinements of this discussion, see [74]. seems that some fine-tuning is necessary to get a small enough slow-roll parameter $\eta$. This $\eta$ problem has been the focus of much recent work (see, for example, $[12,13,92-94]){ }^{7}$ We will, however, simply assume that $\eta \ll 1$ without worrying about whether that assumption requires fine-tuning or not. Our interest will be elsewhere.

The potential provided by $N$ antibranes works out to be [87]

$$
V=\frac{N}{\pi} g_{s}^{3} e^{-12 u} e^{4 A_{\text {inf }}} M_{P}^{4},
$$

where the subscript "inf" indicates for the warp factor at the bottom of the inflationary throat. Henceforth, we will set the number of antibranes to $N=1$ for the following reason: unless the wandering D3 branes are tied together in some way, the last stage of inflation will be described by the dynamics of one D3 brane. Therefore, inflation will end with the annihilation of a single $\mathrm{D} 3 / \overline{\mathrm{D} 3}$ pair, decreasing $V(N)$ to $V(N-1)$. However, $V(N-1)$ is already included in the post-inflationary cosmological constant, so we only consider one of the antibranes as driving inflation.

Relating the potential to the inflationary Hubble scale $V=3 M_{P}^{2} H^{2}$, we find the warp factor to be

$$
e^{A_{\text {inf }}}=(3 \pi)^{1 / 4}\left(\frac{e^{4 u}}{g_{s}}\right)^{3 / 4}\left(\frac{H}{M_{P}}\right)^{1 / 2} \approx \mathcal{O}\left(1-10^{3}\right)\left(\frac{H}{M_{P}}\right)^{1 / 2} .
$$

For values of the Hubble constant

$$
\frac{H}{M_{P}} \approx \mathcal{O}\left(10^{-8}-10^{-5}\right),
$$

the warp factor covers a wide range from $10^{-4}$ (as given in [11]) to nearly unity. Note that (4) gives an interesting bound on the compactification moduli in brane inflation: $e^{4 u} / g_{s} \lesssim\left(M_{P} / H\right)^{2 / 3}$ since $A_{\text {inf }} \leq 0$.

In a sense, this warp factor is comparatively high, in that the effective string mass $1 / \ell_{\text {inf }}=e^{A_{\text {inf }}} / \sqrt{\alpha^{\prime}}$ along with the associated Kaluza-Klein scale $e^{A_{\text {inf }}} / R$ are considerably higher than the inflationary Hubble scale:

$$
\frac{1}{\ell_{\mathrm{inf}}}=\left(12 \pi^{3} g_{s}\right)^{1 / 4}\left(\frac{M_{P}}{H}\right)^{1 / 2} H \gg H .
$$

From the point of view of the effective field theory, this high value for the warp factor is reassuring, since it implies that string and KK corrections will be suppressed. Additionally, it makes sense to think of the antibranes as probes on the compactification manifold. On the other hand, if we are interested in signals from string theory,

\footnotetext{
${ }^{7}$ Within slow-roll inflation it is possible to realize assisted inflation [95-97] with the help of multiple branes [6,21], or with multiple membranes in strongly coupled heterotic $M$ theory [17], or with multiple axions [20], which can ameliorate the supergravity $\eta$ problem to some extent.
} 
having $\ell_{\text {inf }} H \ll 1$ means that corrections to the $\mathrm{CMB}$ are highly suppressed and most likely undetectable. ${ }^{8}$

Brane inflation also has a natural exit; when the branes get within about a string length of the antibranes, a string mode stretched between them becomes tachyonic, resulting in brane annihilation and reheating as in hybrid inflation [100-102]. We will discuss reheating from brane inflation in more detail in Sec. IV.

A related inflationary scenario is the D3/D7 model discussed in $[9,15,103]$. In these models, world volume flux on D7 branes plays the role of antibranes, and the D3 branes are attracted to the D7 branes. In the ending stage of inflation, the D3 brane becomes bound to the D7 brane as an instanton of the world volume flux, and the potential is also of the hybrid inflation type. One advantage of D3/ D7 inflation is that a shift symmetry exists in many cases, which serves to flatten the inflationary potential $[14,104]$.

Another related inflationary mechanism is warped tachyonic inflation, recently proposed by [18]. In this mechanism, a non-Bogomolnyi-Prasad-Sommerfeld (BPS) D brane (presumably wrapping a cycle of the compactification manifold) decays, much as the brane and antibrane annihilate in brane inflation. Localizing the non-BPS brane in a warped region can naturally satisfy slow-roll conditions.

The final mechanism we mention is known as racetrack inflation, which makes use of the nonperturbative potential that stabilizes some of the compactification moduli [16]. The idea is that sufficiently general nonperturbative superpotentials give rise to saddle points in the effective potential, where the slow-roll parameters become small. We should emphasize that the inflaton is a compactification modulus, such as $u$, so the internal space changes throughout inflation. This scenario could possibly lead to nonstandard cosmology during inflation.

\section{Problems with the simple picture}

Let us now return to the SM sector, recalling that we are interested in models in which warping provides some significant contribution to the SM hierarchy. In that case, as we saw above, strings at the tip of the SM throat have an effective mass scale of $1 / \ell_{\mathrm{SM}} \sim M_{\mathrm{SM}}$ (at whatever intermediate scale we choose). Similarly, the Kaluza-Klein mass scale is near $M_{\mathrm{SM}}$. However, during inflation, we know that the SM scale can be much lower than the Hubble scale, $H \sim 10^{13} \mathrm{GeV}$. Even so, the usual approach to inflation in these compactifications is through the 4D effective field theory and naively assumes that the only modification of the compactification geometry is through

\footnotetext{
${ }^{8}$ In brane-antibrane inflation, it was suggested that detectable non-Gaussianities may be produced after the end of inflation due to the tachyonic instability triggered by the open string modes. Such large non-Gaussianity produced can be helpful in constraining not only the string scale but also the string coupling $[98,99]$.
}

the replacement $\eta_{\mu \nu} \rightarrow g_{\mu \nu}^{4}$ of the 4D Minkowski metric with a Friedman-Robertson-Walker (FRW) metric. We can quickly see how such a large Hubble scale leads to inconsistencies in this naive model.

Even at the level of the classical equations of motion, this naive approach already runs into problems. If we consider a 5D Randall-Sundrum model as a proxy for a full 10D compactification, [105,106] have shown that the naive replacement $\eta_{\mu \nu} \rightarrow g_{\mu \nu}^{4}$ breaks down precisely when $H \gtrsim M_{\mathrm{SM}}$. Heuristically speaking, we would expect just such an effect because the KK gravitino masses are $\sim M_{\mathrm{SM}}$, so higher-dimensional gravity should become important as $H \geqslant M_{\mathrm{SM}}$. In particular, the time and space coordinates will have different warping in the extra dimensions, so the 4D Hubble rate will vary over the compactification, which can have interesting cosmological consequences $[107,108]$. The asymmetric warping for the time and space coordinates also leads to a significant violation of Lorentz invariance in the effective field theory. We would expect large corrections to the CMB in that case.

However, there are other, even more drastic corrections to the 10D geometry from string physics. The most straightforward way to see how the SM strings lead to an inconsistency is to consider curvatures. We can already see from a 4D point of view that higher-derivative corrections to gravity will become important because $\ell_{\mathrm{SM}} H \gg 1$. We should, however, look at the 10D curvatures, since we are interested in the compactification geometry. Without evaluating the entire $R^{4}$ correction to IIB supergravity, we note that the Ricci scalar is already large. For a metric of the form (1), the 10D curvature is

$$
\begin{aligned}
R & =12 H^{2} e^{-2 A}+R_{6}-8 \nabla_{6}^{2} A-4\left(\nabla_{6} A\right)^{2} \\
& =12 H^{2} e^{-2 A}+e^{2 A}\left(2 \hat{\nabla}^{2} A+8(\hat{\nabla} A)^{2}\right),
\end{aligned}
$$

where $\nabla_{6}\left(\hat{\nabla}_{6}\right)$ denotes the covariant derivative for the $6 \mathrm{D}$ metric $g_{m n}^{6}\left(\hat{g}_{m n}^{6}\right)$. In the second equality, we have used the fact that $\hat{g}_{m n}^{6}$ is Calabi-Yau (and therefore Ricci-flat). Near the bottom of the SM throat, the curvature is dominated by $H^{2} e^{-2 A}$ and the derivatives of the warp factor are small in comparison. From (4), we have then

$$
R \simeq 12 H^{2} e^{-2 A_{\mathrm{SM}}}=\frac{M_{P}}{3 \pi}\left(\frac{e^{4 u}}{g_{s}}\right)^{-3} e^{4 A_{\mathrm{inf}}-2 A_{\mathrm{SM}}} .
$$

Hence if the SM throat is too strongly warped, the curvature scale will be well above the Planck scale as well as the (10D) fundamental string scale. In this case, the entire tower of higher-derivative corrections should be important. In fact, the tip region of the SM throat would need some sort of nonperturbative string description - a world-sheet conformal field theory (CFT) probably would not be sufficient because the curvature is beyond the 10D Planck scale as well as the string scale. Such high curvatures would also lead to a breakdown of the effective field theory from the $4 \mathrm{D}$ point of view. Let us stress that the EFT does in fact tell 
us that it is breaking down; dimensionally reduction yields Riemann-squared and other higher-derivative terms in the $4 \mathrm{D}$ action. Nor is it clear that an inflationary spacetime would remain a consistent solution of the modified equations of motion.

There is an additional, more subtle way to see that the 4D effective field theory will break down, because, during inflation, the Hubble scale exceeds the effective tension of strings localized at the tip of the SM throat. In particular, any comoving observer will see a prolific creation of strings out of the vacuum during inflation, which is similar to the phenomenon discussed in [109-111]. Heuristically, the calculation is as follows: The Wick rotation of de Sitter spacetime in global coordinates is an $S^{4}$ of radius $1 / H$. In this geometry, there is a world-sheet instanton given by a (Euclidean) string wrapping an equatorial $S^{2}$. The action is just given by product of the area of the $S^{2}, 4 \pi / H^{2}$, and the (effective) tension: $S_{E}=2 / H^{2} \ell_{\mathrm{SM}}^{2}=2 / \alpha^{\prime}\left(H^{2} e^{-2 A_{\mathrm{SM}}}\right)^{-1}$, which, from the discussion around (8), is small in the present situation. Now, the instanton can be Wick rotated back to the Minkowski signature by splitting the $S^{2}$ along an equator. With this analytic continuation, we see that the instanton creates a world sheet filling a $\mathrm{dS}_{2}$ subspace of the original universe. Thus, we can interpret the instanton as creating long strings. The nucleation rate per unit volume of these strings is $e^{-S_{E}}$ (times factors usually of order unity), which is not suppressed. Hence we find the advertised prolific production of strings. In another language, the comoving observer should see a thermal bath at the Gibbons-Hawking temperature $H / 2 \pi$. However, this temperature is well above the effective Hagedorn temperature $T \sim 1 / \ell_{\mathrm{SM}}$ of the strings localized in the SM throat. Hence the thermal bath should be composed largely of long strings (actually, it is likely that there will be many black holes, as well, at such a high temperature).

Of course, all of these difficulties will also arise due to effective strings formed by D-brane wrapping cycles in the compactification if those $\mathrm{D}$ branes are located at the tip of the SM throat. For instance, D1 branes at the tip of the SM throat will also have a low string scale compared to Hubble. For simplicity, though, we will focus on fundamental string physics in this paper.

\section{ALMOST STRING-SCALE INFLATION}

Above, we have found that a naive approach of string inflation leads to inconsistencies for scenarios where the warping differs significantly between the inflationary and SM (or any additional) throats. However, in this section, we will argue that a more sophisticated treatment leads to a picture where the highly warped SM throat becomes essentially "self-repairing" during inflation. We will find a self-consistent picture involving the 4D effective field theory and a modification to the compactification geometry. First, we will examine the consequences of the 4D effective field theory during inflation and interpret them in terms of 10D physics. Then we will see that the emergent 10D picture is still consistent with the 4D effective field theory with controlled $\alpha^{\prime}$ and higher-dimensional corrections. At the same time, we will see how, at least in the correct region of parameter space, those corrections could become observable.

We might note that [112-115] have considered inflationary scenarios with the Hubble scale at or above the string scale, giving rise to an "unstable" phase of strings. Our "self-repairing" scenario is clearly distinct in that we do not encounter any such exotic stringy phase (during inflation).

\section{A. Hubble-induced masses}

To begin, we assume the validity of the effective field theory during inflation, and we will see that it is generically impossible for any scalar to have a mass parametrically lower than the Hubble scale. In the following section, we will then argue that this fact implies that during inflation the SM throat geometry is modified from its true vacuum.

One argument to this effect has been known for some time in supersymmetric models for cosmology [5355,116-118] (see also [119]), which we review here. Consider some supergravity theory with a light scalar $\phi$ (which by abuse of language we will call a modulus), although our argument holds true even for an inflaton. The basic point is that the $\mathcal{N}=1$ supergravity potential

$$
V \propto e^{\mathcal{K}}\left(\mathcal{K}^{i \bar{j}} D_{i} W D_{\bar{J}} \bar{W}-3|W|^{2}\right)+|D|^{2},
$$

contains many cross couplings between any such $\phi$ and the inflaton energy density. For example, assume that $\phi$ has a minimal Kähler potential $|\phi|^{2} / M_{P}^{2}$ and that the inflaton potential comes from the $F$-terms. Then the total potential contains a Hubble-induced mass term of roughly

$$
\frac{1}{M_{P}^{2}}|\phi|^{2} V_{\mathrm{inf}} \approx C H^{2}|\phi|^{2} .
$$

In fact, $[54,55]$ give many possible such Hubble contributions to the effective potential of $\phi$, leading to an effective mass term as above where $|C|=O(1)$ but $C$ may have either sign. If $\phi$ were the inflaton, then it would suffer the supergravity $\eta$ problem during inflation, where $|\eta| \equiv$ $M_{p}^{2} V^{\prime \prime} / V \sim \mathcal{O}(1)$. As it turns out, [11] showed that this type of $\eta$ problem can generalize even to $D$-term inflation, in particular, to the case of brane inflation. In this paper we assume that the $\eta$ problem for the inflaton has been tackled by fine-tuning or some other inflaton-specific mechanism. Fine-tuning could also render the mass of the modulus $\phi$ to be light compared to the Hubble mass $(C \ll 1) .{ }^{9}$ However, we will see below that the percent level fine-tuning that is

\footnotetext{
${ }^{9}$ For a no-scale type Kähler potential $C=0$. At one loop, corrections could be induced, but generically they are small, $|C| \ll 1[120]$.
} 
sufficient for the inflaton $\eta$ problem does not change our story significantly when applied to the modulus $\phi$.

Additionally, we might be interested in a negative Hubble-induced mass, $C<0$, when it is possible to stabilize the modulus at a finite VEV [54,55]. Besides the soft SUSY breaking mass term (10), there could be other contributions from the nonrenormalizable superpotential contribution, i.e., $W \sim \lambda \phi^{d} / M^{d-3}$ with some cutoff scale $M$. Such a contribution would lead to a self interacting potential

$$
|\lambda|^{2} \frac{\phi^{2 d-2}}{M^{2 d-6}}
$$

which can stabilize the modulus to a false vacuum with a finite energy density, where $\lambda \sim \mathcal{O}(1)$ and $d \geq 3$. During inflation the VEV of the modulus will be given by

$$
|\phi| \simeq\left(\frac{C}{(d-1) \lambda} H M^{d-3}\right)^{1 /(d-2)} .
$$

For example, such nonrenormalizable potentials would arise from integrating out the heavy $\mathrm{KK}$ modes. Therefore, the cutoff $M$ would be given by the KaluzaKlein or string scale, which is essentially determined by the behavior of $\phi$. In the end, we expect $M \sim H$ during inflation, so the VEV is bounded by $H$. Additionally, the mass at the new VEV will also be of Hubble scale.

Next we consider a complementary argument that moduli will generically develop Hubble-scale masses during inflation. If our light scalar has a mass $m \leqq H$ in its effective potential, its quantum fluctuations during inflation develop a (steady-state) VEV. Strictly speaking, for a massless scalar in a de Sitter background, the two-point correlation function grows indefinitely for long wavelength fluctuations [121],

$$
\left\langle\phi^{2}\right\rangle \approx \frac{1}{2 \pi^{3}} \int_{H}^{H e^{H t}} d^{3} k\left|\phi_{k}\right|^{2} \approx \frac{H^{3}}{4 \pi^{2}} t .
$$

This result can also be obtained by considering the Brownian motion of the scalar field. For a massive field, one does not obtain an indefinite growth of the variance of the long wavelength fluctuations, but [121-123]

$$
\left\langle\phi^{2}\right\rangle=\frac{3 H^{4}}{8 \pi^{2} m^{2}}\left(1-e^{-\left(2 m^{2} / 3 H\right) t}\right) \rightarrow \frac{3 H^{4}}{8 \pi^{2} m^{2}}
$$

after sufficient e-foldings. In the limiting case when $m \rightarrow$ $H$, the variance goes as $\left\langle\phi^{2}\right\rangle \approx H^{2}$.

Let us now suppose that $\phi$ has self-interactions, including generically a quartic term $\lambda \phi^{4}$. Expanding the action for particlelike fluctuations $\delta \phi$, we find an effective mass term

$$
\frac{1}{2} m_{\mathrm{eff}}^{2} \delta \phi^{2}=\frac{1}{2} m^{2} \delta \phi^{2}+6 \lambda\left\langle\phi^{2}\right\rangle \delta \phi^{2} .
$$

From (14), the contribution from the interaction will in fact dominate the bare mass unless $\lambda<m^{4} / H^{4}$, which is unexpectedly small in our case. Solving (14) for the effective mass, we obtain

$$
m_{\mathrm{eff}}^{2} \approx \frac{3}{\pi} \sqrt{\frac{\lambda}{2}} H^{2}, \quad\left\langle\phi^{2}\right\rangle \approx \frac{H^{2}}{4 \pi \sqrt{2 \lambda}} .
$$

So we see that a self-interaction generates a Hubble-scale induced mass for a light scalar during inflation. This argument holds even if the scalar VEV is shifted by corrections such as (12). Further, this argument generalizes to nonrenormalizable interactions.

We stress that we are not taking the view that the light scalar starts in its true vacuum and then moves away, either due to an induced classical potential or quantum fluctuations. Instead, we imagine that the modulus has some (arbitrary) initial condition during some inflationary phase and then rolls toward a minimum of the potential over a few e-foldings. However, inflationary physics, including quantum fluctuations, keeps $|\phi| \sim H$ over a given patch. The modulus $\phi$ only approaches its true ("bare") ground state after the end of inflation.

Finally, we remind the reader of the potential role in cosmology for such a light scalar. For instance, its presence during inflation typically gives rise to isocurvature fluctuations. If the modulus is sufficiently long lived (longer than the inflaton), then its decay could lead to reheating the universe and the conversion of isocurvature fluctuations into adiabatic density perturbations [124-127]. Within the minimal supersymmetric standard model there are 300 such moduli which can play an important role in cosmology [119]. We will return to reheating from moduli in warped compactification scenarios in Sec. IV B.

\section{B. Ten-dimensional interpretation}

Recall from Sec. II A that the vacuum state geometry of the SM throat suppresses the mass of localized degrees of freedom by a factor of $e^{A_{\mathrm{SM}}} \sim M_{\mathrm{SM}} / M_{P}$. Therefore, since the maximum fundamental mass scale is $M_{P}$, the greatest mass the deformation modulus should take is $M_{\mathrm{SM}}$; this is just the Randall-Sundrum hierarchy. However, by the arguments of Sec. III A above, this modulus should have a mass of approximately $m \sim H$ during inflation if the 4D effective theory is valid. Notice the tension in the two statements. Either the effective field theory must break down, or the SM throat geometry must be modified in some manner consistent with the effective field theory. We assume that inflation does indeed induce a Hubble-scale mass for the SM throat deformation modulus, precisely as in the effective field theory. What we now argue is that the use of the effective field theory is consistent from the 10D point of view and that all the problems of Sec. II C are resolved. ${ }^{10}$

The effective theory itself suggests what happens from the 10D point of view during inflation. In the effective theory, all scalars fluctuate during inflation, which was part

\footnotetext{
${ }^{10}$ We discuss some alternative possibilities in Appendix B.
} 
of our argument for Hubble-scale effective masses. Fluctuations of the SM throat deformation modulus actually cause the throat geometry itself to fluctuate, so we should not expect the SM throat to remain in the vacuum configuration. In fact, the potential for the modulus is asymmetric around its minimum and would tend to push fluctuations toward shorter throat geometries. Additionally, the Kaluza-Klein gravitons, which are also light in the vacuum compactification, should fluctuate as well, further modifying the throat geometry. Unfortunately, we do not have a way to describe the 10D geometry in complete detail. What seems clear, though, is that the warp factor at the tip of the SM throat must be lifted to at least $e^{A_{\mathrm{SM}}} \sim H / M_{P}$. This lifting will allow the deformation modulus to acquire a Hubble-scale mass. The reader should note that this value is still much smaller than the inflationary throat warp factor $e^{A_{\text {inf }}}(4)$, which we assume remains essentially unchanged during inflation.

The short throat with $e^{A_{\mathrm{SM}}} \sim H / M_{P}$ is also consistent with the idea that the compactification should have arbitrary initial conditions. Specifically, the SM throat deformation modulus is complex, with a larger absolute value corresponding to a shorter SM throat. Therefore, it is most likely that the SM throat would start with a large warp factor and then settle into its inflationary state with $e^{A_{\mathrm{SM}}} \sim$ $H / M_{P}$.

Hence we assume that the warp factor lifts up, so the effective mass of the deformation modulus in the SM throat blueshifts to $m^{2}=C H^{2}$, where $C \approx 1$. Since all of the masses relevant for this throat are proportional to the warp factor, the effective string and Kaluza-Klein scales are also raised. To determine the warp factor more precisely, we may further assume that the modulus mass is given by the usual formula for a flux-induced mass. Following the discussion in Appendix A, we then have

$$
e^{A_{\mathrm{SM}}} \approx \frac{\sqrt{C}}{g_{s} n_{f}} e^{3 u \sqrt{\alpha^{\prime}} H}=\frac{\sqrt{C}}{g_{s}^{2} n_{f}} e^{6 u} \frac{H}{M_{P}} .
$$

Note that the second equality uses the fact that a change in the geometry of the SM throat will leave the internal volume (A3) essentially unchanged. Hence, with reasonable values of the parameters (e.g., $g_{s} \sim 1 / 10, n_{f} \sim 10$, $e^{4 u} / g_{s} \sim 10^{3}$ as given in [52]), the warp factor is somewhat larger than the naive estimate above, i.e., $e^{A_{\mathrm{SM}}} \approx$ $10^{4} H / M_{P}$. With (17), we can also estimate the effective string scale in the SM throat during inflation to be ${ }^{11}$

\footnotetext{
${ }^{11}$ In general, the SM throat geometry will vary with time, and so in the following, we will use $\ell_{\mathrm{SM}}$ to denote the instantaneous effective string scale at any given stage of the throat's evolution. However, we will reserve $M_{\mathrm{SM}}$ to denote the effective string mass in the SM throat only when the latter has reached its vacuum configuration.
}

$$
\frac{1}{\ell_{\mathrm{SM}}^{2}} \approx \frac{C e^{6 u}}{n_{f}^{2} g_{s}^{2}} H^{2} \quad \text { or } \quad \ell_{\mathrm{SM}} H \approx \frac{n_{f} g_{s}}{\sqrt{C}} e^{-3 u} \lesssim 1 .
$$

In fact, with the parameter values above, we find $\ell_{\mathrm{SM}} H \approx$ $10^{-1.5}$. Furthermore, if the compactification scale is of the order of the 10D string scale (i.e., $e^{u} \rightarrow 1$ ), we find that $\ell_{\mathrm{SM}} H$ approaches unity. ${ }^{12}$ Similarly the mass scale for KK modes localized in the SM throat is given by

$$
m_{\mathrm{KK}}^{2} \simeq \frac{e^{2 A_{\mathrm{SM}}}}{R^{2}} \approx \frac{C e^{6 u}}{n_{f}^{2} n_{\mathrm{R}} g_{s}^{3}} H^{2}
$$

where the AdS curvature at the tip is roughly $R^{2} \sim$ $g_{s} n_{\mathrm{R}} \alpha^{\prime} \gtrsim \alpha^{\prime}$ in string units - see Appendix A. As usual, the latter inequality ensures that the $\mathrm{KK}$ mass scale is slightly smaller than the effective string scale.

Perhaps the most uncertain assumption above was that the mass of the deformation modulus had the scalings of a typical flux-induced mass. A slightly more conservative estimate would be that the modulus mass corresponds to that of the KK modes localized in the throat. In this case, the hierarchy between the effective string and Hubble scales is $\ell_{\mathrm{SM}} H \approx \sqrt{\alpha^{\prime}} / \sqrt{C} R$. Hence, the separation of scales is independent of the compactification volume and so may be smaller than estimated above. We must require $\sqrt{\alpha^{\prime}} / R<1$ for the SM throat in its ground state in order that supergravity calculations are reliable. As the RR flux determining $R$ is fixed by a quantization condition, one might expect this inequality is maintained in the foreshortened throat produced during inflation.

In any event, either approach seems to yield $\ell_{\mathrm{SM}} H \lesssim 1$. Hence it would seem that the compactification is just within the realm of validity for the 4D effective field theory. Higher-dimensional and high-curvature corrections are somewhat suppressed (and, in fact, the suppression is by discretely tunable parameters), so we have a selfconsistent picture for the SM throat during inflation. That is, the SM throat has some initial condition which is not its true ground state, and, after a few e-foldings, the throat settles into an inflationary state with $e^{A_{\mathrm{SM}}} \sim H / M_{P}$. In fact, this evolution simply mimics the behavior expected for a scalar field during inflation from the point of view of the 4D effective field theory.

Additionally, in the correct range of parameter space, $\ell_{\mathrm{SM}} H$ is not so much less than 1 so that corrections to the CMB may be detectable. In fact, with the parameter values considered above, we expect that higher-dimensional and string theoretic physics may correct the 4D effective theory at about a percent level or even somewhat higher. In terms of the $\mathrm{CMB}$, the corrections may appear as a nonGaussianity (possibly) or, even more likely, as a deviation from scale invariance. However, we leave determining the

\footnotetext{
${ }^{12}$ Also, if derivatives of the warp factor contribute appreciably to the modulus mass, $\ell_{\mathrm{SM}} H$ might be somewhat closer to unity than given in (18).
} 
precise form of the corrections for the future. One direction that may be fruitful to pursue is determining the geometry of the compactification throat in a simplified model; as indicated in $[105,106]$, the time and noncompact space directions will have warp factors that differ by $\mathcal{O}\left(\ell_{\mathrm{SM}} H\right)$ corrections, and perhaps a modified cosmology can be extracted from the accompanying violation of Lorentz invariance.

\section{REHEATING OF STANDARD MODEL THROAT}

During inflation, the inflaton dominates the energy density of the universe, and any radiation or matter density redshifts to negligible values very quickly. How the energy density ends up in SM degrees of freedom after inflation is the question of reheating, and it seems a particularly challenging problem in brane inflation models, where the inflationary and SM branes can be separated both geometrically and by large potential barriers (due to the warp factor). Fortunately, the inflationary and SM throats can communicate [128,129], and the SM can reheat [56]. In this section, we will review the reheating mechanism of [56] and give a more detailed calculation of the reheating rate and final reheat temperature of the SM throat. ${ }^{13}$ Then we discuss the dynamics of the SM throat itself during the era following inflation and comment on what effects those dynamics can have on reheating. Based on our reheating calculations, we will find that strings will likely have an important role in reheating the light SM fields. The key point is that, in a wide range of parameter space, the SM sector reheating temperature (as calculated in EFT) is higher than the Hagedorn temperature of the SM strings. We turn to the cosmology of those strings in the next section.

\section{A. Reheating from inflationary throat}

Reheating begins in the inflationary throat with tachyon condensation when the brane/antibrane pairs are within a string length of each other $[5-7,100]$. Because the tachyon couples to the brane gauge fields, its condensate actually breaks the gauge groups on the branes as in the Higgs mechanism. The rolling of the tachyon also excites the gauge fields on any remaining branes along with massive closed strings. One of the attractive features is, of course, the formation of cosmic F and D strings by the Kibble mechanism, as widely discussed in the literature $[10,130]$; their stability (and its model dependence) has been discussed in [131,132]. In any case, we expect that much of the inflationary energy density will end up as gravitational modes on a short time scale. Here, we will review the argument given by [56]. Reheating proceeds in a multistage process, and we will examine each step. Finally, we will give an estimate of the reheating temperature and

\footnotetext{
${ }^{13}$ See also the recent discussion in $[57,58]$.
}

energy density. In the following, we will use $H_{\star}$ to denote the Hubble parameter at the end of inflation. ${ }^{14}$

We would also like to note that reheating itself can lead to constraints on brane inflation models, although our focus is on the final reheat temperature. The papers [56-58] have considered graviton production during reheating, and [57] has additionally addressed the production of other dangerous relics, such as long-lived KK modes in the inflationary throat.

\section{Brane annihilation}

As we noted in Sec. II B, brane inflation is a stringy realization of hybrid inflation. When the brane and antibrane come within a string length, an open string mode becomes tachyonic, and the slow-roll conditions fail. Condensation of the tachyon annihilates the branes, which creates massive closed strings. Although a homogeneous calculation for D3 branes does not lead to efficient reheating, tachyon modes with nonzero momentum can lead to more efficient decay channels [133]. In that case, the closed strings have typical mass $m \sim 1 / \ell_{\text {inf }} g_{s}$ and nonrelativistic transverse momentum $p_{T} \sim 1 / \ell_{\text {inf } \sqrt{g_{s}}}$, where again the subscript inf indicates the value at the bottom of the inflationary throat. This intrinsically stringy annihilation has a time scale of roughly $\ell_{\text {inf }}$, so the total energy density stored in the closed string sector is of the order of $\tau \sim 1 / \ell_{\text {inf }}^{4} g_{s}$, the brane tension. ${ }^{15}$

We also note that the tachyonic instability can also excite nonlinear gravitational fluctuations, giving rise to significant non-Gaussianity in the $\mathrm{CMB}$, which is constrained by present data from WMAP. In some situations, these constraints put limits on the inflationary throat string scale [98], although the cases we consider are probably safe because $\ell_{\text {inf }} H_{\star} \ll 1$.

\section{Tunneling rate}

To calculate the rate of decay from massive closed strings to KK modes with significant wave functions in the SM throat, it is helpful to think of this decay as a two step process. First, we note that the massive closed strings, being nonrelativistic in the compact directions, will be localized near the tip of the inflationary throat at the time they decay. Therefore, this decay should occur at the local string time scale $\ell_{\text {inf }}$ and should result in KK gravitons localized in the inflationary throat. The next step is then the transfer of these gravitons to the SM throat by tunneling, as has been discussed in [56] - see related discussion in $[57,58]$.

Actually, this tunneling rate has been considered earlier in $[128,129]$, which discuss the tunneling of localized

\footnotetext{
${ }^{14} \mathrm{We}$ discuss possible generalizations of this mechanism and reheating in other string embeddings of inflation in Appendix C.

${ }^{15}$ See also [134] for a review and further references.
} 
gravitons from one AdS throat to another. Those papers give a tunneling rate

$$
\Gamma_{t}=\frac{\pi^{2} n^{4}}{16} e^{5 A_{\text {inf }}} \frac{1}{R},
$$

where $R$ is the curvature scale of the initial throat (and by assumption, of the final throat) and $n$ is the KK mode number of the graviton (measured in the initial throat). $R$ is actually an effective anti-de Sitter radius, which is $R \sim$ $\sqrt{g_{s} n_{\mathrm{R}} \alpha^{\prime}}$ (see Appendix A for the definition of $n_{R}$ ) and $A_{\text {inf }}$ is given by (4). In terms of the inflationary Hubble scale $H_{\star}$, the rate is

$$
\Gamma_{t} \approx 3 \pi^{2}\left(\frac{n}{2}\right)^{4}\left(\frac{12 \pi^{7}}{g_{s} n_{\mathrm{R}}^{2}}\right)^{1 / 4}\left(\frac{e^{4 u}}{g_{s}}\right)^{3}\left(\frac{H_{\star}}{M_{P}}\right)^{3 / 2} H_{\star} .
$$

Taking $n_{R} \sim 10-10^{2}, n=1$, and our usual values $\left(e^{4 u} / g_{s}\right) \sim 1-10^{3}$ and $g_{s} \sim 1 / 10$, we find that $\Gamma_{t}$ ranges over $\mathcal{O}\left(10-10^{10}\right)\left(H_{\star} / M_{P}\right)^{3 / 2} H_{\star}$, largely due to the strong dependence on $e^{4 u} / g_{s}$.

Because the rate (21) is very sensitive to the KK mode number, we should also check that $n \sim 1$ is a reasonable assumption. We know that the compact momentum of each KK graviton is $p_{T}=f / \ell_{\text {inf }} \sqrt{g_{s}}$ with $f \sim 1 / 2$ just from the transverse momentum of the massive closed strings. However, the mass (and transverse momentum) are quantized approximately in units of $1 / \ell_{\mathrm{inf}} \sqrt{g_{s}} \overline{\mathrm{R}}_{\mathrm{R}}$. Therefore, the typical KK mode number is $n=f \sqrt{n_{\mathrm{R}}}$. For $n_{\mathrm{R}} \sim 10-100$, we have at most $n \sim 5$. Additionally, the KK modes can interact with each other and decay into lighter KK states with a time scale of order $\ell_{\text {inf }}$. This decay competes with tunneling, so we expect some reduction in $n$ for the typical tunneling rate. Therefore, it seems reasonable to take a small typical mode number $n \lesssim 3$.

There is another effect, which we have not calculated, which could possibly suppress the tunneling rate further. In the 5-dimensional models used to approximate the KK mode wave functions and the tunneling rate between throats, the two distinct throats have the same AdS curvature scale. However, in the string compactifications we study, the different throats have different AdS curvatures. If the curvature in the SM throat (at the appropriate value of the warp factor) is larger than in the inflationary throat radius, then the tunneling rate would be suppressed [135]. However, we will assume that the suppression is not great enough to reduce the rate much below our estimate (21).

For completeness, we can also give the tunneling rate in terms of the inflationary throat string scale, which is the form of the tunneling rate given in [56]:

$$
\Gamma_{t}=\pi^{2}\left(\frac{n}{2}\right)^{4}\left(\frac{1}{g_{s} n_{\mathrm{R}}}\right)^{1 / 2} e^{4 A_{\mathrm{inf}}} \frac{1}{\ell_{\mathrm{inf}}} .
$$

This is heavily suppressed with respect to the string scale, so the total decay rate for the decay of massive string modes to SM throat KK modes is determined by this tunneling rate. Comparing this rate to the decay rate into massless 4D gravitons imposes strong restrictions on constructing a viable model - see archive version of [56].

It may be that considering the tunneling process with a more sophisticated model reduces this suppression (22) and eases these restrictions. For example, the potential barrier of a cascading geometry is smaller [135] than in the simple AdS model of $[128,129]$. Another possibility comes from the introduction of D7 branes in the SM construction, as was briefly mentioned at the end of Sec. II A. The purely D7 brane open strings have KK scale masses and are weakly coupled to the SM fields. However, they may still provide an interesting channel for tunneling if the D7 brane ventures out from the SM throat to the inflationary throat.

\section{Excitation of SM brane modes}

The ultimate stage of warped reheating is excitation of the SM brane degrees of freedom, which effectively traps energy density in the SM sector. Since the SM branes lie at the bottom of the SM throat, we expect that the decay of the KK modes into light SM brane modes will occur over a time $\ell_{\mathrm{SM}}$, the string scale at the tip of the SM throat. The key issue, then, is the value of $\ell_{\mathrm{SM}}$ during reheating; after all, during inflation, $1 / \ell_{\mathrm{SM}} \geq H_{\star}$, while the geometry should settle to its vacuum state $1 / \ell_{\mathrm{SM}} \sim M_{\mathrm{SM}}$ long after inflation. As we argue in Sec. IV B 1, we expect that the time dependent string scale is about the Hubble scale at that time, or $1 / \ell_{\mathrm{SM}}(t) \sim H(t)$. Further, the time scale for energy to reach the SM throat is the decay rate (21), so we expect the string scale at reheating to be $1 /\left.\ell_{\mathrm{SM}}\right|_{\text {reheat }} \sim \Gamma_{t}$, unless $\Gamma_{t} \lesssim M_{\mathrm{SM}}$, in which case $1 / \ell_{\mathrm{SM}} \sim M_{\mathrm{SM}}$. In either case, we expect the brane excitation rate $\Gamma_{\mathrm{SM}} \gtrsim \Gamma_{t}$.

However, the above discussion assumes that the KK gravitons interact perturbatively with the branes. If there is any coherence in the KK modes, however, semiclassical effects can be important. In particular, there is evidence that bulk modes can reheat the SM brane through parametric resonance [136,137]. Parametric resonance, or preheating, can be efficient and rapid [138-141], occurring over a time scale set by oscillations of the KK gravitons. In our case, this time scale is shorter than $\ell_{\mathrm{SM}}$. Even so, the thermalization time scale for SM brane modes would be around $\ell_{\mathrm{SM}}$ (at least in the effective field theory), so we end up with the same conclusion as the purely perturbative interaction.

\section{4. $S M$ reheat temperature}

The final stage of reheating is thermalization of the SM degrees of freedom. In general, there is a stage with a reheating rate $\Gamma<H(t)$, so the SM modes will redshift appreciably before thermalizing, and we can consider the 
reheating to occur when $\Gamma \sim H(t)$. We have argued that the reheating rate is determined by the tunneling rate, so $\Gamma \sim$ $\Gamma_{t}$. Assuming that there is instantaneous thermalization of the decay products, the reheat temperature comes out to $\mathrm{be}^{16}$

$$
T_{r h} \sim 0.1 \sqrt{\Gamma \bar{M}_{P}} \sim \sqrt{\Gamma_{t} \bar{M}_{P}},
$$

which follows by comparing the thermal energy density $\sim T^{4}$ (for SM radiation) to the energy driving the expansion $\sim H(t)^{2} M_{P}^{2}$.

There are two cases we should consider: If $\Gamma_{t} \gtrsim$ $M_{\mathrm{SM}}^{2} / M_{P}$, the reheat temperature is larger than $M_{\mathrm{SM}}$ (at the time of reheating) - one finds that this is a plausible situation combining (18) and (21). In this case, the thermal bath is sufficiently hot enough to excite fundamental strings (and perhaps D strings). This means that the estimate (23) is unreliable, since it depends on field theoretic relations between the temperature and energy density. Instead, we should have a truly string theoretic reheating process. In that case, thermalization should result in a gas of long open strings in the Hagedorn regime $\beta \sim \ell_{\mathrm{SM}}$ and energy density near $\sim H(t)^{2} M_{P}^{2} \sim \Gamma_{t}^{2} M_{P}^{2}$. (Again, we are simply assuming that the energy driving the expansion of the universe is dominated by the open strings.) At temperatures above $M_{\mathrm{SM}}$ we would also excite the KK modes in the SM throat. However, they need not be in thermal equilibrium with the SM degrees of freedom because the KK modes do not share the same charges as the SM. For example, KK modes with high angular momentum in the compact directions (as in [57]) may be localized in the SM throat away from the SM branes, so they may only be weakly coupled to the SM degrees of freedom. It would, of course, be interesting to describe the string reheating process in more detail. We will discuss the long open string phase and its cosmological evolution in Sec. V.

The second case is that $M_{\mathrm{SM}}$ is relatively large and $\Gamma_{t}$ is small so that $T_{r h} \lesssim M_{\mathrm{SM}}$. In that case, the field theoretic reheating calculation will be valid, and only SM radiation (and no open strings or KK modes) will be excited.

\section{B. Reheating from modulus decay}

In Sec. III, we described how the deformation modulus should lift the warp factor in the SM throat during inflation so that $\ell_{\mathrm{SM}} H_{\star} \lesssim 1$. Our field theoretic expectations are

\footnotetext{
${ }^{16} \mathrm{~A}$ full thermalization in the field theory case need not be completed during reheating [142], especially in the case of supersymmetric theories, because $2 \leftrightarrow 2$ and $2 \rightarrow 3$ processes, required for thermalization [143], are mediated via gauge bosons/gauginos and Higgses. They obtain large masses from the VEVs of the flat directions of the minimal supersymmetric SM, which slows down the rate of thermalization. It is plausible to define a temperature corresponding to the kinetic equilibrium despite the lack of full thermal equilibrium; in our case we will treat $T_{r h}$ as a symbolic temperature which would help us compare the decay rates from various processes.
}

then that the modulus obtains a VEV of order $H_{\star}$ and contributes to the energy density with $\rho \sim H_{\star}^{4}$. Of course, as the Hubble parameter evolves during the last few efoldings of inflation and reheating, so should the modulus in the SM throat. Here, we will discuss possible scenarios for the modulus evolution and the consequences for cosmology.

\section{SM throat evolution}

There are two distinct possibilities, associated with two possibilities for the Hubble-induced potentials discussed in Sec. III A. Simply put, the two possibilities correspond to whether the VEV of the modulus is induced by cross couplings in the potential or by quantum fluctuations, as described in Sec. III A.

The case of cross couplings, in which the potential develops a tachyonic mass $m_{\mathrm{eff}}^{2} \sim-H^{2}$ near $\phi=0$, is of importance phenomenologically for the Polonyi problem [53] and baryogenesis [54,55], and [55] has already studied the behavior of a modulus with such an induced potential, so we will review their conclusions. Let $\phi_{0}(H)$ be the Hubble-induced ground state (12) for the given value of the Hubble constant. Since the potential is induced by the energy density, it will remain throughout reheating, and $\phi(t)$ will track $\phi_{0}(H(t))$ until $H(t) \sim M_{\mathrm{SM}}$ for a matterdominated epoch. (It is a simple calculation to show that their argument carries over to the radiation-dominated case, as well.) Therefore, we expect that the modulus $\phi$ should not begin oscillating around its true vacuum until $H(t) \sim M_{\mathrm{SM}}$, which is when the bare potential should become important - we expect the bare or vacuum mass of the modulus to be closer to the KK scale but there should only be a small separation between these scales. During the oscillating phase, preheating and perturbative reheating will proceed as usual, except that the modulus will not necessarily dominate the energy density. As a function of time, this motivates us to expect that $\ell_{\mathrm{SM}}(t) H(t) \sim 1$ for $H(t) \gtrsim M_{\mathrm{SM}}$.

In the second case where quantum fluctuations dominate the VEV, $\langle\phi\rangle=0$ over many horizons but $\left\langle|\phi|^{2}\right\rangle \neq 0$. In fact, because of the two-point expectation, $\phi$ has a coherent phase and magnitude $|\phi| \sim H$ over horizon distances $1 / H_{\star}$ at the end of inflation. In this case, we expect that $\phi$ will begin to oscillate around its true vacuum relatively quickly. However, the magnitude of the oscillation decays as $1 / t \propto H(t)$ (disregarding reheating), and we might expect that the SM throat warp factor only decays with the magnitude of the oscillation, so $\ell_{\mathrm{SM}}(t) H(t) \sim 1$ for $H(t) \gtrsim$ $M_{\mathrm{SM}}$. Once again, preheating and reheating will proceed as normal.

Before proceeding, let us note two things. First, we should take the preceding discussion of the modulus (and SM throat) evolution with a grain of salt. After all, it would not be surprising if there are 10D or string corrections, and we do not have a complete picture of the stationary ge- 
ometry during inflation. Still, we expect that the effective field theory gives us a reasonable qualitative picture, given that the corrections are somewhat suppressed during inflation. Additionally, when $H(t) \lesssim M_{\mathrm{SM}}$, we expect the SM throat to settle into its true vacuum, so we at least understand the endpoint of its evolution. The other point is that we do not expect the SM throat modulus to make a dominant contribution to the energy density initially. The modulus might eventually dominate the energy density if it decays slowly enough to SM fields (because the energy density in the modulus redshifts at least as slowly as matter). This is reminiscent of the cosmological moduli problem, and we will explore this point below.

\section{Reheat temperature from modulus}

Once the modulus oscillates coherently [roughly when the Hubble parameter is less than the modulus mass $H(t) \lesssim$ $\left.m_{\phi}\right]$, the oscillations mimic a pressureless fluid, whose energy density redshifts as $a^{-3}$, where $a$ is the scale factor. During this oscillation, the modulus can decay nonperturbatively. This is due to the fact that every time the modulus passes through its minimum $\phi=0$, the velocity of the field changes sign and vacuum fluctuations are enhanced due to nonadiabatic evolution. This triggers the fragmentation of the homogeneous condensate, known as preheating [138-141].

Although this initial stage of particle creation could be explosive, preheating does not lead to a complete thermalization. Thermalization is achieved via decay of the condensate (assuming that preheating does not use all the energy of the condensate). If the effective mass of the decay product is heavier than the mass of the modulus, $g \phi>m_{\phi}$, where $g$ is some gauge or Yukawa coupling, the modulus could decay into light SM particles only through loop diagrams involving heavy particles, with an effective coupling of the type $\left(g^{2} /\langle\phi\rangle\right) \phi \bar{\psi} \gamma^{0} \partial_{0} \psi$, where $\psi$ is a light fermion [144]. On the other hand, the modulus will decay at tree level if $g \phi<m_{\phi}$. Thus, the decay rate is given by

$$
\Gamma_{\phi} \approx \begin{cases}\frac{g^{4} m_{\phi}^{3}}{\phi^{2}} & \left(g \phi>m_{\phi}\right), \\ \frac{g^{2} m_{\phi}}{8 \pi} & \left(g \phi<m_{\phi}\right) .\end{cases}
$$

We are interested in the possibility that decay of the modulus $\phi$ is the final stage of reheating, so we assume that $\phi$ dominates the energy density when it decays. ${ }^{17}$ Then $H \sim m_{\phi} \phi / M_{P}$, so the amplitude of the modulus and Hubble parameter during the decay are given by $[124,125]$

\footnotetext{
${ }^{17}$ After all, if $\phi$ is subdominant, its decay will just increase the energy density in the dominant component.
}

$$
\phi_{d} \approx\left\{\begin{array}{l}
\left(g^{4} m_{\phi}^{2} M_{P}\right)^{1 / 3}, \quad H_{d} \approx\left\{\begin{array}{l}
g^{4 / 3}\left(\frac{m_{\phi}}{M_{P}}\right)^{2 / 3} m_{\phi}, \\
g^{2} M_{P},
\end{array} g^{2} m_{\phi} .\right.
\end{array}\right.
$$

Depending on the mass of the modulus there are two distinct possibilities. If the compactification has $m_{\phi} \sim$ $M_{\mathrm{SM}}$ (which is the natural region of parameter space to expect), the energy density stored in the modulus at the time of decay is $\rho_{\phi} \sim M_{\mathrm{SM}}^{2} \phi_{d}^{2}$. Assuming rapid thermalization of the decay products, the reheat temperature is

$$
T_{r h} \approx 0.1 \sqrt{\Gamma_{d} M_{P}} \approx\left\{\begin{array}{l}
0.1 g^{2 / 3} \sqrt{M_{\mathrm{SM}}^{5 / 3} M_{P}^{1 / 3}}, \\
0.1 g \sqrt{M_{\mathrm{SM}} M_{P}}
\end{array}\right.
$$

In the first case (large $\phi_{d}$ and decay by loops), this temperature is higher than $1 / \ell_{\mathrm{SM}} \sim M_{\mathrm{SM}}$ as long as $g$ is not too much less than 1 , so the field theoretic approximation will break down, and there will be some string theoretic phase. Then the final stage of reheating will be given by the decay of long open strings into radiation, as described in the next section. In the second case, we derive $g^{3} M_{P} \lesssim M_{\mathrm{SM}}$ using (25). This then implies that the reheat temperature is above the string scale $M_{\mathrm{SM}}$ if the coupling is in the range $\sqrt{M_{\mathrm{SM}} / M_{P}} \lesssim g \lesssim\left(M_{\mathrm{SM}} / M_{P}\right)^{1 / 3}$ for which the final stage of reheating will also be described by the decay of open strings. If the coupling is $g \lesssim \sqrt{M_{\mathrm{SM}} / \bar{M}_{P}}$, the modulus will reheat the SM directly to radiation. In fact, since we expect a stringy phase when the density is $\rho \gtrsim M_{\mathrm{SM}}^{4}$ or equivalently $H \gtrsim M_{\mathrm{SM}}^{2} / M_{P}$, the Hubble parameter at modulus decay (25) is exactly consistent with this expectation.

When might the SM throat modulus come to dominate the energy density? During the modulus evolution, its energy density should redshift no more quickly than matter (it redshifts like matter during oscillation and somewhat more slowly if it tracks a changing potential). Therefore, we expect that the modulus will most likely come to dominate the energy density if the other component is radiation. In other words, if the tunneling rate (21) is low enough that the inflationary sector would reheat the SM throat directly to radiation, the modulus could very well end up dominating the energy density. Another option is that the modulus could decay by loops before tunneling occurs, in which case the inflationary sector would still dominate the energy density, but the modulus could reheat the SM sector to a long open string phase.

\section{Cosmological consequences of the modulus}

We will give a few examples of possible consequences of the SM throat modulus decay (once again, we remind the reader that we do not have a complete picture of the throat dynamics).

Clearly, one consequence is related to the fact that the roll of the SM throat modulus is a necessarily higherdimensional effect. In terms of a simple Randall- 
Sundrum model, we would interpret it as the roll of the radion field. Since the 4D FRW equations are modified when the radion is not stabilized, it would seem that this era may have some form of nonstandard cosmology.

Additionally, the decay of the modulus might be responsible for generating adiabatic density perturbations in the CMB $[124,125,145,146]$. Such a scalar can generate isocurvature fluctuations in the CMB (although, since $m_{\phi} \sim$ $H_{\star}$ during inflation, the large scale fluctuations may be suppressed). In our case the modulus is not likely to dominate the energy density during its coherent oscillations, if there were already a gas of open strings present, which redshift like $a^{-3}$ in equilibrium and $a^{-2}$ out of equilibrium. However, if there were no open strings created prior to the decay of the modulus or the modulus decays after the strings decay to radiation, then the modulus could convert all its isocurvature fluctuations into adiabatic modes, which is known as the curvaton mechanism (see [145]). However, this issue is rather model dependent, and, depending on various parameters, a favorable model can be created along the lines of $[124,125,146]$.

Let us consider what happens if the modulus decays while the energy density of the universe is dominated by an open string phase of density $\rho_{o}$. This would lead to a partial conversion of the isocurvature fluctuations to the adiabatic fluctuations and also a significant nonGaussianity parameter $[145]^{18}$

$$
f_{N L} \geq \frac{1}{r} \simeq \frac{1}{g^{4 / 3}}\left(\frac{\mu^{2}}{M_{\mathrm{SM}}^{4}}\right)\left(\frac{M_{\mathrm{SM}}}{M_{P}}\right)^{2 / 3}
$$

for the perturbations sourced by $\phi$. Here, $r$ is the ratio between the energy densities of the modulus and the strings at the time of decay, $r=M_{\mathrm{SM}}^{2} \phi_{d}^{2} / \rho_{o}$. We have assumed that the modulus decays by loops before the strings convert to radiation, and (to be conservative) we took the smallest value of $\rho_{o} \sim \mu^{2}$ for effective string tension $\mu$. Obviously, once we assume that the modulus obtains a Hubbleinduced mass correction during inflation, the perturbations of the modulus will be suppressed on large scales during inflation and therefore the higher-order perturbations as well. The non-Gaussian signature may then be undetectable by the forthcoming CMB experiments. A possible enhancement in non-Gaussianity $f_{N L} \sim 50$ may arise naturally if the modulus mass is smaller than the inflationary Hubble scale, $m_{\phi} \leq H_{\text {inf }}$, and it decays nonperturbatively into the light degrees of freedom [147].

\footnotetext{
${ }^{18}$ The non-Gaussianity parameter is defined by the Bardeen potential which arises while studying the temperature anisotropy $\Phi=\Phi_{\text {Gauss }}+f_{N L} \Phi_{\text {Gauss. }}^{2}$ In the curvaton scenario $\Phi=$ $-(r / 5)\left(\delta \rho_{\phi} / \rho_{\phi}\right)$. In the case of the modulus $\delta \rho_{\phi} / \rho_{\phi}=$ $2(\delta \phi / \phi)+(\delta \phi / \phi)^{2}$. This leads to the non-Gaussianity parameter $f_{N L} \sim 5 / 4 r[145]$.
}

In some models, the modulus decay could also directly produce weakly interacting massive particles (WIMPs). For example, one of the best known supersymmetric particles is the gravitino, which the modulus could produce. Then there are known cosmological consequences. For a $\mathrm{TeV}$ mass gravitino the decay time extends through big bang nucleosynthesis, so therefore the initial abundance has to be small, i.e., $n_{3} / s<10^{-10}$. The direct decay of the modulus into gravitinos, however, generates significant abundance [148]. This would then require heavy gravitinos above $50 \mathrm{TeV}$, which would decay before big bang nucleosynthesis. However, if the gravitinos decay after the freezeout temperature of thermally generated neutralinos, then their decay would also dilute the cold dark matter abundance. Detailed model building issues have been studied in [149]. The models we discuss might avoid these problems entirely, also; the supersymmetry breaking scale is not tied to the warping, so the gravitino could be heavier than the modulus.

\section{REHEATING DUE TO OPEN STRING DECAY}

In this section, we will discuss reheating of SM modes (i.e., the massless modes on the SM branes) through the decay of long open strings on the SM branes. Since we do not have a full understanding of the SM throat geometry while $M_{\mathrm{SM}}<H<H_{\star}$, we pick up the story when $H \sim$ $M_{\mathrm{SM}}$, when we expect the SM throat to settle into its true vacuum geometry, which we reviewed in Sec. II A. Why bring in long open strings in this regime? We remind the reader that the effective field theory reheating temperature (23) can be sufficiently high that string modes should have been excited during reheating, so there may be some stringy phase of evolution. In addition, even with $H \sim$ $M_{\mathrm{SM}}$, the $4 \mathrm{D}$ energy density $\rho \sim M_{\mathrm{SM}}^{2} M_{P}^{2}$ is much greater than the effective string density $\rho_{0} \sim M_{\mathrm{SM}}^{4} \sim 1 / \ell_{\mathrm{SM}}^{4}$. Therefore, we still expect a long string phase, and we will focus on fundamental strings for simplicity. ${ }^{19}$

Our goal is to establish a basic framework for the cosmology of open strings, which we will see can be somewhat different than the standard cosmology of (closed) cosmic strings. This framework would apply in many potential situations in string cosmology beyond our scenario-whenever open strings are a significant source of energy density. In particular, we could imagine brane inflation with low-energy supersymmetry, so that the standard model could live on whatever branes survive the brane-antibrane annihilation. In that case, we might expect tachyon condensation to produce long open strings on the surviving branes, which could provide at least some component of reheating in the standard model.

To discuss reheating through long open strings, we first must describe the distribution of open strings as well as the

\footnotetext{
${ }^{19}$ We discuss the alternate possibility of the SM throat developing a black-brane horizon in footnote ${ }^{20}$ and Appendix D 3.
} 
possible decay modes and decay rates of the long strings. Once we have reviewed that physics, we will include the decay modes in the cosmological evolution of the open string gas and discuss reheating from open strings to massless SM modes.

\section{A. String thermodynamics with $D$ branes}

In this section, we review essential features of the thermodynamics of open strings, with more details in Appendix D 1. We emphasize that this discussion is relevant for any discussion of open strings in cosmology and not just our particular model.

Many authors have studied thermodynamics of open strings in the background of D branes [59-61], with the D branes essentially considered as static objects. Intrinsic in our use of these results is the assumption that we can at least approximately trust flat spacetime string thermodynamics in cosmology when $H \lesssim 1 / \ell_{\mathrm{SM}}$. All the papers [59-61] agree that open strings dominate closed strings if the number density of $\mathrm{D}$ branes (in the transverse volume) is greater than unity in string units, so the open string thermodynamics is independent of closed string thermodynamics. Therefore, we relegate a discussion of closed string thermodynamics to Appendix D 2. Although D strings or other wrapped D branes might enter into thermal equilibrium at high densities, we will leave that question for future work and focus on a pure string gas for simplicity. ${ }^{20}$

One quantity we will need is the distribution of string number per unit string length per volume parallel to the $\mathrm{D}$ branes. Specializing to D3 branes, this is [59]

$$
\mathcal{D}(\ell)=\frac{2 a N_{D}^{2}}{b V_{\perp}} e^{-\ell / L}, \quad L=\frac{1}{\mu\left(\beta-\beta_{H}\right)},
$$

where $L$ is the average length of the open strings, $\mu$ is the string tension, and $\beta\left(\beta_{H}\right)$ is the inverse (Hagedorn) temperature. In the above, both $a, b$ are proportional to $g_{s}$ (with a ratio $b / a \sim \mu$ ), $N_{D}$ is the number of branes, and $V_{\|}, V_{\perp}$ are, respectively, the volumes along and transverse to the $\mathrm{D}$ branes. For now, we are working in 10D units for ease of comparison to the literature, and throughout we take the thermodynamic limit $E \propto V_{\|} \rightarrow \infty$. Because it appears in the distribution, $V_{\perp}$ is an important parameter in the thermodynamics. This volume is not the full volume of the compactification manifold because the warp factor in the SM throat acts as a potential for the compact position; rather we can treat $V_{\perp}$ as the volume accessible to the strings through their thermal and quantum fluctuations, as

\footnotetext{
${ }^{20}$ It is common string theory folk-knowledge that a Hagedorn gas of strings undergoes a phase transition to a black hole or black brane (i.e., something with a horizon). In Appendix D 3, we will argue that the situation is more subtle. Though we cannot rule out the appearance of a horizon, we expect the appearance of a long string phase.
}

in [132]. ${ }^{21}$ It turns out that we can approximate $V_{\perp} /\left(4 \pi^{2} \alpha^{\prime}\right)^{3} \sim 1$; the calculation is given in detail in Appendix D 4.

If we want to add D7 branes to the SM throat, (28) is rearranged somewhat, and $a / b$ takes a different dimensionality. However, in the end, the prefactor of the exponential takes the same order of magnitude. Also, each type of string $\left(33,77\right.$, or 37) has its own distribution with $N_{D}^{2} \rightarrow$ $N_{3}^{2}, N_{7}^{2}, N_{3} N_{7}$, respectively. In this paper, we will work mostly to order of magnitude and simply write $N_{D}^{2}$.

So far, we have worked in ten-dimensional units, but we can translate to $4 \mathrm{D}$ effective units for the purpose of cosmology. First, note that the 4D energy density is given by $\rho=\int d \ell \mu \ell \mathcal{D}(\ell)$. Then using (28), we can relate the average string length to this density,

$$
\begin{aligned}
\bar{\ell} & =e^{-A_{\mathrm{SM}}} L=\frac{1}{N_{D}}\left(\frac{V_{\perp}}{\left(4 \pi^{2} \alpha^{\prime}\right)^{3}}\right)^{1 / 2}\left(\frac{\left(2 \pi \ell_{\mathrm{SM}}\right)^{6} \pi b}{a} \frac{\rho}{M_{\mathrm{SM}}^{2}}\right)^{1 / 2} \\
& \approx\left(\frac{\rho}{N_{D}^{2} M_{\mathrm{SM}}^{4}}\right)^{1 / 2} \ell_{\mathrm{SM}},
\end{aligned}
$$

where we have substituted $\mu=M_{\mathrm{SM}}^{2} / 2 \pi$, the effective SM string tension. Note that the average string length is longer than the string length for $\rho \gtrsim N_{D}^{2} M_{\mathrm{SM}}^{4}$, which is the minimum density we consider. Henceforth, all quantities are in 4D units (i.e., measured in terms of the effective SM string scale) except for $V_{\perp}$ and explicit factors of $\alpha^{\prime}$. Let us also make explicit that $\overline{\bar{\ell}}$ (and other lengths) are not the lengths projected onto the $4 \mathrm{D}$ spacetime but rather the total length in four-dimensional units. However, a simple random-walk argument shows that $\bar{\ell}$ and the projected length differ only by factors of order unity.

Since we are also interested in the cosmological evolution of the open string gas, we would like to know the equation of state. Because the compact directions are stabilized, we just need the pressure in the noncompact directions, which is approximately

$$
p=\frac{1}{\beta}\left(\frac{4 \pi a}{b} \frac{N_{D}^{2}}{M_{\mathrm{SM}}^{2}} \frac{\rho}{V_{\perp}}\right)^{1 / 2} \approx N_{D} M_{\mathrm{SM}}^{2} \sqrt{\rho}
$$

in the Hagedorn regime [150-152]. This equation of state is somewhat unusual in cosmology, but, in a wide range of interest, we will see that the open strings behave like a pressureless gas.

A critical question for cosmology is when the open strings are in equilibrium. The gas of strings can remain in thermodynamic equilibrium as long as a typical string interacts once per Hubble time (see the discussion of equilibration times in [59]). Fortunately, the probability for two strings to interact given that they cross is known to be $[132,153]$

\footnotetext{
${ }^{21}$ This procedure is somewhat ad hoc; a proper treatment would include the potential in the statistical description of the strings from the outset.
} 


$$
P=g_{s}^{2}\left[\frac{\left(1-\cos \theta \sqrt{1-v^{2}}\right)^{2}}{8 v \sin \theta \sqrt{1-v^{2}}}\right] \frac{\left(4 \pi^{2} \alpha^{\prime}\right)^{3}}{V_{\perp}} .
$$

Following typical arguments about cosmic strings [154], we know that the empty area surrounding a string in a network of density $\rho$ is $\mu / \rho$. This leads to a crossing rate per unit length of string of $v \rho / \mu$ and an interaction rate of

$$
\frac{d \Gamma}{d \ell}=g_{s}^{2}\left[\frac{\left(1-\cos \theta \sqrt{1-v^{2}}\right)^{2}}{8 \sin \theta \sqrt{1-v^{2}}}\right] \frac{\left(4 \pi^{2} \alpha^{\prime}\right)^{3}}{V_{\perp}} \frac{\rho}{\mu} .
$$

To get the interaction rate for a typical string, we just multiply by the average string length as given above. Then the equilibration rate is

$$
\Gamma \approx \frac{N_{D}^{2} g_{s}^{2}}{\ell_{\mathrm{SM}}}\left(\frac{\rho}{N_{D}^{2} M_{\mathrm{SM}}^{4}}\right)^{3 / 2}
$$

averaging over angles and velocities, in agreement with the rough discussion of [59], and again substituting $\mu=$ $M_{\mathrm{SM}}^{2} / 2 \pi$. Of course, if the distribution of open strings starts out far from equilibrium, we should use the typical length scale of that distribution, rather than $\bar{\ell}$, to determine the equilibration rate.

Note that the dependence for (33) on the density of open strings means that it is very difficult for the strings to fall out of equilibrium. Suppose that the open strings dominate the universe, so $H \sim \sqrt{\rho} / M_{P}$. Then a comparison gives $\Gamma / H \sim N_{D} g_{s}^{2}\left(M_{P} / M_{\mathrm{SM}}\right)\left(\rho / N_{D}^{2} M_{\mathrm{SM}}^{4}\right)$. As long as $\bar{\ell} \gtrsim \ell_{\mathrm{SM}}$, the strings will remain in equilibrium. In fact, it seems that the open strings will maintain equilibrium unless they either start out very far from equilibrium or are subdominant.

Finally, we should contrast these results to the usual intuition developed from the study of cosmic strings, which is that long cosmic strings enter a scaling solution rather than maintain equilibrium (see [154], for example). The difference arises because of the different distribution of strings. For cosmic (closed) strings at typical densities, the gas of strings is composed of many small loops and a few very long strings. As a consequence, two short loops cannot join to form a long string, and, additionally, short strings will not often intersect long strings. However, long strings will intersect each other roughly once a Hubble time, so they can decay by emission of short loops relatively efficiently. The long string population cannot regain energy as quickly as it loses energy, so the strings fall out of equilibrium. In contrast, open strings are distributed uniformly up to a length of roughly $\bar{\ell}$, above which the population decreases. Therefore, there is a sizable population of intermediate length strings, which can join with each other to form long strings. Therefore, the entire string gas can remain in equilibrium if $\Gamma \geq H$.

\section{B. Decay rates of long open strings}

Since the reheating of the SM throat seems likely to lead to a phase of long open strings, a crucial ingredient is the decay of those strings. Long open string decay has been the subject of multiple studies, mostly in flat backgrounds with what we would now recognize as D9 branes (or D25 branes in the bosonic case) [155-158]. The relevant calculations of total decay rates for open strings on superstring $\mathrm{D}$ branes of arbitrary dimensionality appear in [62]; we review the results here. Even though these decay rates are for static spacetimes, we will assume their validity since the Hubble parameter is $H(t) \lesssim M_{\mathrm{SM}}$. Additionally, these decay rates only apply, strictly speaking, to leading Regge trajectory states, but we will use them for the entire open string network.

Since the D3 branes do not fill the entire 10D spacetime, the decay rate depends on the polarization of the open string in question, longitudinal or transverse to the branes. Realistically, there is a wide variety (a "discretuum," to borrow the term) of decay rates due to the many possible polarizations of a long string, but we will lump all the strings into two classes, those longitudinally polarized and those transversely polarized. In fact, these are precisely the two cases considered by [62].

The decay of a string polarized longitudinally to a D3 brane is nearly a splitting rate per unit length, as found in [62]. For our purposes, we restore factors of $g_{s}$ and write the string oscillator number $N$ in terms of the string's length $\ell, 2$ finding

$$
\Gamma_{\text {split }}=\frac{(2 \pi)^{3}}{2 \sqrt{2}} \frac{N_{D} g_{s}}{\ell_{\mathrm{SM}}}\left(\frac{\ell}{\ell_{\mathrm{SM}}}\right)\left[\frac{1}{\pi} \ln \left(\frac{\ell}{4 \ell_{\mathrm{SM}}}\right)\right]^{-3} .
$$

We have inserted an additional factor of $N_{D}$ compared to [62] because the string can split on any of the D3 branes. Note that the log factors suppress this rate compared to a decay rate per unit length; physically this means that the string prefers slightly to split near its ends. These logarithm factors are caused by quantum mechanical fluctuations of the string away from the world volume of the D3 brane. For now, the important thing to note is that even a longitudinally polarized string accesses the transverse (compact) dimensions with a distance scale that grows logarithmically in the length of the string. Finally, we should note that splitting has been included in the analysis of open string equilibrium, even though it is not the dominant interaction (see [59]).

On the other hand, transversely polarized strings decay only by radiating massless strings from their end points [62] with a rate

\footnotetext{
${ }^{22}$ The conversion is $\sqrt{N}=\ell / 4 \ell_{\mathrm{SM}}$, which follows from (for example) the light-cone Hamiltonian for a string configuration $X^{1}=(\ell / 2) \cos \sigma \cos \omega \tau, \quad X^{2}=(\ell / 2) \cos \sigma \sin \omega \tau, \quad 0 \leq \sigma \leq \pi$, $\ell \omega=2$. This is explained in [156]. The calculation turns out to be the same for a transversely polarized string rotating in two transverse directions.
} 


$$
\Gamma_{\mathrm{rad}}=\frac{\sqrt{2}(2 \pi)^{4}}{8} \frac{N_{D} g_{s}}{\ell} .
$$

Note that completely transversely polarized strings do not really have any length in the noncompact dimensions. We assume, though, that a typical "transversely" polarized string has about the same length of string along the brane directions as it does transverse to them. This is the same assumption we made in the thermodynamics. The reader should also be aware that this decay rate for the leading Regge trajectory strings is slower than expected for a typical transversely polarized string. However, as long as a string has any significant transverse polarization, it will only decay to SM modes by radiation from its endpoints, and we take the rate (35).

The key issue is to determine whether the long strings are longitudinally or transversely polarized. Let us quickly work through the case of strings in thermal equilibrium near the Hagedorn temperature. Our rationale is as follows: if the linear scale of quantum fluctuations of a longitudinal string is as great as the linear scale of thermal fluctuations, then the strings can be considered to be longitudinally polarized. Otherwise, the strings are transversely polarized. Since the Hagedorn strings can always access three of the dimensions of the SM throat tip, then we take the thermal length scale to be $L / 2 \pi \sqrt{\alpha^{\prime}}=\left(g_{s} n_{\mathrm{R}}\right)^{1 / 2} /(4 \pi)^{1 / 3}$, as follows from our expression for $V_{\perp}$ (16). The quantum length scale we can extract from the decay rate (34) is $L / 2 \pi \sqrt{\alpha^{\prime}}=\left[\ln \left(\ell / 4 \ell_{\mathrm{SM}}\right) / \pi\right]^{1 / 2}$. Therefore, we expect that open strings will be longitudinally polarized only for lengths longer than some critical value,

$$
\ell \gtrsim \ell_{c} \equiv 4 \ell_{\mathrm{SM}} \exp \left[\left(\frac{\pi}{16}\right)^{1 / 3} g_{s} n_{\mathrm{R}}\right] \approx 10^{3} \ell_{\mathrm{SM}} .
$$

The numerical value is given for $g_{s} n_{\mathrm{R}} \sim 10$. For strings at equilibrium, there will only be many longitudinally polarized strings if $\bar{\ell} \gtrsim 10^{3} \ell_{\mathrm{SM}}$, which would require $\rho \gtrsim$ $10^{6} N_{D}^{2} M_{\mathrm{SM}}^{4}$. (We should mention that different compactification models will give different formulas for $\ell_{c}$.)

\section{Evolution of string network and reheating}

Here we will discuss the transfer of energy density from long open strings into radiation.

\section{Strings in equilibrium}

For specificity, we will begin with the case of equilibrium open strings, giving a rough analysis of reheating into radiation. $^{23}$

\footnotetext{
${ }^{23}$ Throughout, we will assume that, by this stage of reheating, the energy density in $\mathrm{KK}$ modes is subdominant. If any $\mathrm{KK}$ modes are in equilibrium, they are a gas at temperature $T \sim$ $M_{\mathrm{SM}}$; nonequilibrium KK modes decay with a rate $\dot{\rho} \propto \rho_{\mathrm{KK}}$, which is much faster than (41) below for $\rho_{\mathrm{KK}} \gtrsim \rho_{o}$ (unless the KK modes are nearly stable).
}

If we start by ignoring the decay of open strings to radiation, the continuity equation, with the pressure (30), is

$$
\dot{\rho}+3 \frac{\dot{a}}{a}\left(\rho+N_{D} M_{\mathrm{SM}}^{2} \rho^{1 / 2}\right)=0,
$$

which has the solution

$$
\rho=\left[\left(\rho_{0}^{1 / 2}+N_{D} M_{\mathrm{SM}}^{2}\right)\left(\frac{a_{0}}{a}\right)^{3 / 2}-N_{D} M_{\mathrm{SM}}^{2}\right]^{2} .
$$

Here the subscript 0 indicates an initial value. Note that for densities much larger than $N_{D}^{2} M_{\mathrm{SM}}^{4}$, the strings redshift as if they are pressureless, which is not surprising given that the pressure grows more slowly than linearly with density. The deviation from this matterlike redshifting is of order $N_{D} M_{\mathrm{SM}}^{2} / \rho^{1 / 2}$. We should remember, however, that, as this reaches order unity, the strings will become an equilibrium bath of radiation. Therefore, we will mostly consider the open string gas to be pressureless.

Next we will consider the energy loss to radiation by transversely polarized strings (remember that splitting of longitudinally polarized strings is accounted for by the thermodynamics). The rate of density loss is simply written as

$$
\left.\dot{\rho}\right|_{\mathrm{rad}}=\int_{\ell_{\mathrm{SM}}}^{\ell_{c}} d \ell \mathcal{D}(\ell) \Gamma_{\mathrm{rad}}(\ell) \Delta E(\ell)
$$

where $\Delta E(\ell)$ is the energy lost by a string of length $\ell$ in each radiative decay $\left[\mathcal{D}(\ell)\right.$ and $\Gamma_{\text {rad }}(\ell)$ are given, respectively, by (28) and (35)]. We can calculate $\Delta E$ by noting that the radiation process decreases the level of a transversely polarized string by 1 [62]. Working in the center of mass frame, $E^{2}=N / \ell_{\mathrm{SM}}^{2}$, so

$$
\Delta E(\ell)=-\left(2 \sqrt{N} \ell_{\mathrm{SM}}\right)^{-1} \approx-2 / \ell,
$$

using our identification of $N=\left(\ell / 4 \ell_{\mathrm{SM}}\right)^{2}$. This estimate assumes that the string is long enough to neglect recoil and that relativistic energy shifts of the emitted radiation averages out. The lower limit on the integral comes from insisting that the strings be long, and the upper limit comes from the requirement that they be transversely polarized.

Putting everything together, we find

$$
\begin{aligned}
\left.\dot{\rho}\right|_{\mathrm{rad}}(\bar{\ell}) & \approx-\frac{\left(N_{D} g_{s}\right)\left(N_{D}^{2} M_{\mathrm{SM}}^{4}\right)}{\bar{\ell}} \Gamma\left(-1 ; \ell_{\mathrm{SM}} / \bar{\ell}, \ell_{c} / \bar{\ell}\right) \\
& \approx-\frac{\left(N_{D} g_{s}\right)\left(N_{D}^{2} M_{\mathrm{SM}}^{4}\right)}{\ell_{\mathrm{SM}}}
\end{aligned}
$$

is given by an incomplete gamma function. The final approximation is appropriate for $\bar{\ell} \gg \ell_{\mathrm{SM}}$, which we will assume throughout. All told, the SM sector energy in radiation and open strings obeys the equations 


$$
\begin{gathered}
\dot{\rho}_{o}+3 \frac{\dot{a}}{a} \rho_{o}+\frac{\left(N_{D} g_{s}\right)\left(N_{D}^{2} M_{\mathrm{SM}}^{4}\right)}{\ell_{\mathrm{SM}}}=0, \\
\dot{\rho}_{r}+4 \frac{\dot{a}}{a} \rho_{r}-\frac{\left(N_{D} g_{s}\right)\left(N_{D}^{2} M_{\mathrm{SM}}^{4}\right)}{\ell_{\mathrm{SM}}}=0 .
\end{gathered}
$$

These equations can be solved analytically; taking $A=$ $2\left(N_{D} g_{s}\right)\left(N_{D}^{2} M_{\mathrm{SM}}^{4}\right) / \ell_{\mathrm{SM}}$, we find

$$
\begin{aligned}
& \rho_{o}=-\frac{2 A}{9 H}+C_{1}\left(\frac{a_{0}}{a}\right)^{3}, \\
& \rho_{r}=\frac{2 A}{11 H}+C_{2}\left(\frac{a_{0}}{a}\right)^{4}, \text { for } \rho_{o}>\rho_{r} \\
& \rho_{o}=-\frac{A}{5 H}+D_{1}\left(\frac{a_{0}}{a}\right)^{3}, \\
& \rho_{r}=\frac{A}{6 H}+D_{2}\left(\frac{a_{0}}{a}\right)^{4} \text { for } \rho_{o}<\rho_{r},
\end{aligned}
$$

where $a_{0}$ is the initial scale factor for the given era.

We can use (43) and (44) to get a qualitative picture of reheating by open strings. Imagine that we take our initial conditions to be $H \sim M_{\mathrm{SM}}$ and $\rho_{r}$ negligible. Then the initial phase is dominated by the strings, $C_{1} \sim M_{P}^{2} M_{\mathrm{SM}}^{2}$, and the equality between strings and radiation occurs at about $H_{\text {eq }} \sim N_{D} g_{s}^{1 / 3}\left(M_{\mathrm{SM}} / M_{P}\right)^{2 / 3} M_{\mathrm{SM}}$. Following into the radiation era, reheating ends when $\rho_{o} \sim 0$, which turns out to be at $H_{f} \lesssim H_{\text {eq }}$. In the end, we find a final radiation density of $\rho_{r} \lesssim\left(g_{s} M_{P} / M_{\mathrm{SM}}\right)^{2 / 3} N_{D}^{2} M_{\mathrm{SM}}^{4}$. This result is a bit larger than the typical string density $M_{\mathrm{SM}}^{4}$, so a more detailed analysis of energy transfer between strings and radiation is clearly necessary. However, it seems likely that the final reheat density and temperature for SM radiation will be around $\rho \sim M_{\mathrm{SM}}^{4}$ and $T \sim M_{\mathrm{SM}}$, respectively. ${ }^{24}$

\section{Nonequilibrium strings}

In this section, we will give a few comments about the evolution of nonequilibrium strings, for which we do not $a$ priori know the distribution of lengths. As we have seen in

\footnotetext{
${ }^{24} \mathrm{As} M_{\mathrm{SM}}$ is a free parameter in our discussion, we note that the above result raises various phenomenological challenges if $M_{\mathrm{SM}}$ is too low. If $M_{\mathrm{SM}} \sim \mathcal{O}(1 \mathrm{TeV})$ then the most important problem will be to obtain the observed baryonic asymmetry $n_{B} / n_{\gamma} \sim$ $6.1 \times 10^{-10}[1]$; because of the larger parameter space, electroweak baryogenesis in minimal supersymmetric SM, in principle, has a much better chance to succeed. However, there are a number of important constraints, and lately Higgs searches at CERN LEP have narrowed down the parameter space to the point where it has all but disappeared $[159,160]$. However for an intermediate scale $M_{\mathrm{SM}}$ baryogenesis could take place via other means [119]. The other challenge will be to obtain the right abundance for the cold dark matter. Below $M_{\mathrm{SM}} \sim 1 \mathrm{TeV}$ the only viable candidate is the coherent oscillations of the axionic cold dark matter [161-163]. For an intermediate string scale it is possible to have a viable KK cold dark matter whose abundance could be either obtained nonperturbatively from the decay of the modulus, or from thermal excitations.
}

the equilibrium case, the string distribution was important in the rate of decay to radiation; we suspect that a nonequilibrium string distribution would be just as important. With that in mind, we can give only a few general comments.

Out of equilibrium, we must distinguish two populations of open strings, superhorizon $(\ell>1 / H)$ and subhorizon $(\ell<1 / H)$. While superhorizon strings grow with the cosmological expansion, the subhorizon strings can only decay. In addition, since the strings interact rarely, there is an effective maximum to the length of any given string, due to the splitting of longitudinal polarizations. That is, as any string approaches $\ell_{c}$ defined in (36), the SM branes come within the quantum radius of the string, so the string can split with a string-scale rate per unit length (treating the logarithm as roughly constant near $\ell_{c}$ ). Therefore, the longitudinal strings rapidly break into smaller strings, so there should be a large population of superhorizon strings only while $\ell_{c}$ is larger than the Hubble radius. (There may be a few strings with atypically large deviations from the SM branes, and these few strings could be transversely polarized but longer than $\ell_{c}$.)

Let us consider the superhorizon strings a little more carefully. Since the open strings are out of equilibrium, they are not interacting even once a Hubble time, so they cannot enter a scaling solution. In that case, the superhorizon strings could enter a stringlike phase, redshifting as $a^{-2}$, at least for $\ell_{c} \gtrsim 1 / H$. Then, as a given string stretches to a length of $\ell_{c}$, it will split, so the distribution of strings will evolve; numerical simulations will be necessary to understand the evolving distribution.

Finally, we note that nearly all the nonequilibrium strings should decay to radiation on a time scale given roughly by $\ell_{c}$ for two reasons. First, the radiative decay rate (35), for typical strings, is roughly $\Gamma \gtrsim 1 / \ell_{c}$. Second, by $t \sim \ell_{c}$, typical transversely polarized strings will be subhorizon length, so they will no longer stretch and will simply decay. Only a few atypically long transversely polarized strings should survive beyond $t \sim \ell_{c}$. Additionally, these should eventually become subhorizon or longitudinally polarized and decay as well. Since $H \sim$ $1 / \ell_{c}$ occurs much before nucleosynthesis, it seems that light element abundances are protected in this case.

\section{DISCUSSION: POSSIBLE SIGNATURES}

Finally, in this section, we will speculate on the possible cosmological signatures of the light SM sector strings in these warped models. The discussion will be ordered chronologically, from inflation onward.

\section{A. Fluctuations in inflation}

As we mentioned in the Introduction, high-energy physics can alter the inflaton fluctuation spectrum at the earliest stages of inflation [22-47]. So far, in the absence of a theory of quantum gravity or a specific realization of 
inflation in string theory, the effects of "trans-Planckian" physics on the inflaton have been modeled by modified high-energy dispersion relations, modified initial conditions (vacua) for the inflaton, and irrelevant operators in the inflation EFT. The key point is that, depending on the type of signals expected, $H / M \gtrsim 1 / 10$ (or $H^{2} / M^{2} \gtrsim$ $1 / 10$ ) are necessary for corrections to the usual spectrum to be observable [22]. Here, we have argued that a significantly warped SM throat will naturally provide $H / M \sim$ $\mathcal{O}\left(10^{-2}-1\right)$ during inflation - see Eq. (18). Here, $M$ may represent either the effective Kaluza-Klein or string scales in the SM throat. Hence it is likely that higher-dimensional and stringy physics may be observable.

In fact, we can estimate the strength of the corrections. One of the (leading) $\mathcal{O}\left(\alpha^{\prime}\right)^{3}$ corrections to the supergravity action $[164,165]$ contains a term (up to prefactors)

$$
R^{\alpha \beta \mu \nu} R_{\lambda \rho \mu \nu} R_{\alpha}{ }^{m n \lambda} R^{\rho}{ }_{m n \beta} \approx R^{\mu \nu \lambda \rho} R_{\mu \nu \lambda \rho}\left(\nabla_{m} \nabla_{n} A\right)^{2},
$$

where the right-hand side follows by conformally rescaling the 10D metric. Furthermore, $R^{\mu \nu \lambda \rho} R_{\mu \nu \lambda \rho} \sim e^{-4 A} H^{4}$ (plus terms depending on derivatives of the warp factor) by counting powers of the warp factor. If we dimensionally reduce this term, the contribution to the action is

$$
\begin{aligned}
\delta S & \approx M_{10}^{8} \alpha^{\prime 3} \int d^{4} x \sqrt{-g_{4}} \int d^{6} x \sqrt{g_{6}} H^{4}\left(\nabla_{m} \nabla_{n} A\right)^{2} \\
& \approx M_{P}^{2} \alpha^{\prime 3} H^{4} \int d^{4} x \sqrt{-g_{4}}\left\langle e^{-2 A}\left(\nabla_{m} \nabla_{n} A\right)^{2}\right\rangle,
\end{aligned}
$$

where

$$
\langle\cdots\rangle \equiv \frac{1}{V_{w}} \int d^{6} x \sqrt{g_{6}} e^{2 A} \cdots=\frac{1}{V_{w}} \int d^{6} x \sqrt{\hat{g}_{6}} e^{-4 A} \cdots
$$

is the average over the compact manifold, weighted by the same power of the warp factor that gives the warped volume. Since the warp factor is very small near the tip of the SM throat, this region dominates the average. Therefore, we evaluate the average there; by using Riemann normal coordinates, we can estimate $\left(\nabla_{m} \nabla_{n} A\right)^{2} \sim\left(g_{s} n_{\mathrm{R}} \alpha^{\prime}\right)^{-2}$. In the end, we find

$$
\delta S \approx \frac{\left(M_{P} H\right)^{2}}{\left(g_{s} n_{\mathrm{R}}\right)^{2}} \frac{H^{2}}{M_{\mathrm{SM}}^{2}} .
$$

This is suppressed only by $\left(g_{s} n_{\mathrm{R}} \alpha^{\prime}\right)^{-2}\left(H / M_{\mathrm{SM}}\right)^{2}$ compared to the Einstein-Hilbert term, so we expect that it may be detectable.

Distinguishing our string compactification effects from field theoretic models will likely be difficult, however. One suggestion is to examine, order by order in $H / M$, the higher-dimension operators generated by string theory and KK physics. If some characteristic pattern of coefficients for these higher-derivative terms emerges (or better yet, if some of the coefficients are forced to vanish by a symmetry), this pattern may be used to single out compactification physics as the source of any signal. The work of [166] regarding inflaton fluctuations in higher-derivative gravity may be useful for determining the actual CMB spectrum in such a case. In a similar spirit, $[48,49,167]$ have given a characteristic pattern of modifications to the CMB that might arise in brane inflation due to noncanonical inflaton kinetic terms. For another approach, relying on post-inflation evolution, see [168].

One should also consider the possibility of inherently stringy effects during inflation. While the light SM sector strings have $\ell_{\mathrm{SM}}$ less than the Hubble length $1 / H$, we do not expect the separation of scales to be very large. Hence, for example, the creation of long strings, as discussed at the end of Sec. II C, is suppressed but not enormously. So one might find a moderate production of strings during inflation. There might also be unusual quantum effects coming from macroscopic virtual strings during inflation or fluctuations of the string field. Again the point is that the separation of $\ell_{\mathrm{SM}}$ and $1 / H$ should not be immense. So perhaps virtual processes can modify the quantum behavior of the inflaton with nonlocal or stringy effects. While necessarily vague, it may be that string field theory will be necessary to develop these ideas.

\section{B. Nonstandard cosmology during reheating}

We also noted that, for $H_{\star} \gtrsim H(t) \gtrsim M_{\mathrm{SM}}$, the SM throat geometry will evolve from its inflationary state to the true ground state geometry. In Sec. IV B, we modeled this evolution in terms of EFT by discussing the qualitative behavior of the SM throat deformation modulus. It is worth reiterating the point that, in a Randall-Sundrum model of the compactification, the evolution of the SM throat would be modeled by a rolling of the radion. What changes to standard FRW cosmology might result is an interesting question for future work; it seems likely that there would be some corrections for the following reason. As explained in $[169,170]$, the usual Friedmann equation is modified in a Randall-Sundrum universe if the radion is not stabilized; essentially, this fact is because the Einstein equations are five dimensional. Therefore, we might guess that, if the radion is rolling in its potential (that is, it is not completely stabilized by its potential) due to some finite energy density, the Friedmann equation will be corrected. Additionally, in solving the higher-dimensional equations of motion, the space and time directions may develop different warp factors, leading to a violation of Lorentz invariance (see the solutions of $[105,106]$, for example).

\section{Early structure formation (primordial black holes)}

We have argued that the universe is dominated by a gas of long open strings with negligible pressure after the end of inflation. Therefore, the curvature fluctuations created during inflation enter the Hubble radius and perturb the long open strings. As is well known, inside the Hubble 
radius, the linear perturbations in cold dark matter grow, see [171]:

$$
\delta(t)=\delta\left(t_{i}\right)\left(\frac{t}{t_{i}}\right)^{2 / 3},
$$

where $\delta$ is the density contrast and the time $t_{i}$ is chosen to normalize the perturbations entering the Hubble radius. This is analogous to the Jean's instability in a static universe, but expansion slows the exponential growth in the density contrast to a power law. The overdense regions expand slowly, reach a maximum radius, contract, and eventually virialize to form a bound nonlinear system with a density contrast of $\delta \sim \mathcal{O}(1)$.

There are many possibilities for these gravitationally bound systems which one can entertain, such as objects made up of SM baryons, the earliest stars. Nevertheless, as the long strings decay into radiation, the most tenacious objects would be, of course, black holes (which might be the only structures to survive). These primordial black holes [172] can survive until the present, unless their Hawking radiation is important. The lifetime of an evaporating black hole is given by

$$
\frac{\tau}{10^{17} \mathrm{sec}} \simeq\left(\frac{M}{10^{15} \text { grams }}\right)^{3} .
$$

Note that a black hole of initial mass $M \sim 10^{15} \mathrm{~g}$ will evaporate at the present epoch while $M \sim 10^{9} \mathrm{~g}$ will evaporate around the time of nucleosynthesis. Assuming that we form black holes with approximately the mass contained in the horizon (for a review see [173]), we have

$$
M_{\mathrm{HOR}} \approx 10^{9} g\left(\frac{\left(10^{11} \mathrm{GeV}\right)^{4}}{\rho_{o}}\right)^{1 / 2} \text { for } \rho_{o}>N_{D}^{2} M_{\mathrm{SM}}^{4} .
$$

If the string-dominated phase lasts until $\rho_{o} \sim\left(10^{8} \mathrm{GeV}\right)^{4}$, black holes with mass $M \sim 10^{15} \mathrm{~g}$ are formed and can live through today. In any case, massive black holes above $10^{9} \mathrm{~g}$ are astrophysically interesting. Depending on their present abundances, they can be safe with distinct astrophysical signatures. For example, black holes with mass $M \sim 5 \times 10^{14} \mathrm{~g}$ are already constrained from the current $\gamma$-ray bursts as long as the fraction of the black hole energy density to the energy density of everything else is $\alpha_{\text {evap }}<$ $10^{-26} M / M_{P}$ [174]. In the extreme case that $M_{\mathrm{SM}} \sim$ $1 \mathrm{TeV}$, the string-dominated phase lasts until the electroweak phase, and black holes of mass $M \sim 10^{25} \mathrm{~g}$ can be formed. They would live through the present; it would be interesting to see if there are observational constraints on such black holes.

\section{Gravitational waves}

There are also several possibilities for the generation of gravitational waves (beyond those created as fluctuations during inflation).
One possibility occurs because the transition from inflation to a long string-dominated phase is not instantaneous. There is a brief period of radiation which, however, quickly becomes subdominant. During these transitions there would be a slight bump in the gravitational wave spectrum which would have a frequency cutoff roughly given by the geometric mean of two scales: $\omega=$ $2 \pi a(t r) H_{t r} / a(\tau) \approx 2 \pi \sqrt{H_{0} \bar{H}_{t r}}$, possibly allowing the detectability of such gravitational waves by future experiments.

Another possibility is that the long strings themselves source gravitational waves. Clearly, during the long open string phase, there will be a subdominant long closed string component (see Appendix D 2), and these closed strings will eventually decay into gravitons. Additionally, long open strings can dynamically develop cusps, just as closed strings, which then produce beams of gravity waves (see [154] for the appropriate cosmic string literature).

\section{E. Other effects}

The prolonged phase of matter (open string) domination has other mild effects in cosmology. For instance, the required number of e-foldings during inflation could be considerably smaller by

$$
N(k)=62-\frac{1}{12} \ln \frac{V_{\text {end }}}{N_{D}^{2} M_{\mathrm{SM}}^{4}} .
$$

For $V_{\text {end }} \sim 10^{64}(\mathrm{GeV})^{4}$ and $N_{D}^{2} M_{\mathrm{SM}}^{4} \sim 10^{12}(\mathrm{GeV})^{4}$, the number of e-foldings required to explain the observed CMB spectrum and the flatness and the homogeneity problem is only 52 e-foldings.

Additionally, as [57] has pointed out, KK modes with large compact angular momentum in warped throats can have very long lifetimes because light (and SM) modes do not carry the same angular momentum on the internal space. Since either reheating from the inflationary sector or the SM throat modulus can populate these KK modes, we have a natural mechanism for production of relics. What remains to be determined is whether and at what density these KK relics freeze out. Another possible candidate relic is a leading Regge trajectory open string polarized transverse to the SM brane. However, since the open string gas stays in equilibrium very easily, we would need some nonthermal production process for these strings.

A matter-dominated period, either from open strings or oscillations of the SM throat modulus, could also serve to dilute otherwise overly abundant gravity waves. As pointed out in [56], a low tunneling rate could allow long strings or $\mathrm{KK}$ modes in the inflationary throat to decay into massless gravitons. Fortunately, a large enough energy density in matter, either from the modulus or open strings, could dilute the gravitons sufficiently to avoid later problems. ${ }^{25}$

\footnotetext{
${ }^{25}$ We thank J. Cline for discussions on this point.
} 
Finally, we mention that the long open string gas near the Hagedorn temperature suffers from large thermal fluctuations (in the canonical ensemble). ${ }^{26}$ Therefore, the density and temperature should vary from Hubble region to Hubble region (and even within Hubble regions). If these fluctuations can survive being washed out by the subsequent radiation phase, they could leave a significant imprint on the CMB.

\section{SUMMARY}

To summarize, we have considered string inflation in the context of warped compactifications with multiple throats. In scenarios where the warping differs significantly between throats (such as in scenarios where the inflationary scale is much higher than the SM scale), the standard approach of treating inflation as a perturbation on the ground state configuration is inconsistent. Not only would the 4D EFT break down due to KK mode excitations, but the supergravity approximation would also break down due to large 10D curvatures. Instead, we argued that the vacuum geometry of highly warped regions [roughly $e^{A_{\mathrm{SM}}} \lesssim e^{2 A_{\text {inf }}}$ - see (8)] is modified during inflation. Our argument was based on intuition from 4D EFT, and we looked for a consistent picture of the physics. As it turned out, the string (and KK) mass scale is just above the Hubble scale, or $\ell_{\mathrm{SM}} H \lesssim 1$, in the modified geometry. The effective field theory is then (just) consistent, but there may be corrections from KK and string physics, which can lead to potentially observable perturbations of the CMB.

Also, we analyzed reheating of the SM sector, following the "warped reheating" scenario of [56]. We also stress that we are taking the view that the compactification is never in its vacuum until well after inflation ends. The energy stored in the SM throat itself then and the relaxation of the geometry to its "ground state" may have interesting implications for reheating, as discussed in Sec. IV B. In both cases, we saw that there is a strong possibility for reheating to create a phase of long open strings in the SM throat. We provided a rough analysis of the open string phase based on thermal equilibrium, finding that the strings redshift like matter. Additionally, the strings slowly decay to SM radiation, eventually leaving a radiation bath at about the Hagedorn temperature $M_{\mathrm{SM}}$. Standard hot big bang cosmology can proceed as normal from that point.

Finally, in the previous section, we listed some possible observational consequences and directions for future research. It will be very important to analyze these possible signals, especially to see if there is some characteristic signature of string theory or $\mathrm{KK}$ physics, nonGaussianity and inhomogeneous reheating due to strings,

\footnotetext{
${ }^{26}$ Independently, in [175] the authors are studying thermal fluctuations of a string ensemble in the context of noninflationary cosmology. We thank R. Brandenberger for bringing their work to our attention.
}

generating $\mathrm{CMB}$ anisotropy due to string fluctuations during the Hagedorn phase, relic KK particles, and the gravity wave signals from various transitions in the early universe.

Certainly our preliminary investigation leaves many open questions. The most pressing amongst these is perhaps to find a more precise description of the SM throat geometry during inflation. While this is undoubtedly a technically challenging question, our arguments indicate that it should be within the reach of supergravity calculations. In any event, the issues raised here should be a strong precaution for the "modular" approach to model building where separate throats are introduced to produce apparently separate scales for, e.g., inflation, SM physics, and the cosmological constant.

\section{ACKNOWLEDGMENTS}

We would like to thank O. Aharony, R. Brandenberger, A. Buchel, C. Burgess, X.-G. Chen, J. Cline, S. Hofmann, S. Kachru, L. Kofman, J. Polchinski, M. Savage, and M. Schulz for useful comments and conversations. The work of A.R. F. was supported by a John A. McCone Fellowship in Theoretical Physics at the California Institute of Technology. A.R.F. received additional partial support from DOE Grant No. DE-FG03-92-ER40701. Research at the Perimeter Institute is supported in part by funds from NSERC of Canada and MEDT of Ontario. R. C. M. is further supported by an NSERC Discovery grant. A. R. F. and A.M. would also like to thank the Aspen Center for Physics for hospitality during the final stages of this work.

\section{APPENDIX A: CONVENTIONS AND NOTATION}

Here we will describe conventions and notation used throughout the paper.

We start by describing the relation of the various fundamental scales of the compactification. The 10D string frame gravitational action is

$$
S=\frac{1}{2(2 \pi)^{7} \alpha^{14}} \int d^{10} x \sqrt{-g} e^{-2 \varphi} R+\cdots,
$$

where $\varphi$ is the dilaton. Since we assume throughout the paper that the dilaton (as well as the volume modulus of the compactification) is stabilized at a high-energy scale, we separate it into a VEV and a fluctuation, $\varphi=\ln g_{s}+\delta \varphi$. Then the background value of the Einstein-Hilbert term prefactor is $M_{10}^{8} / 2=1 / 2(2 \pi)^{7} \alpha^{\prime 4} g_{s}^{2}$. The $10 \mathrm{D}$ Planck constant is slightly larger than the fundamental string scale in the perturbative limit, i.e., $\alpha^{\prime} M_{10}^{2} \gtrsim 1$.

In the dimensional reduction, the zero mode $h_{\mu \nu}$ of the graviton has a wave function given by the warp factor, $\delta g_{\mu \nu}=e^{2 A} h_{\mu \nu}$, where $\delta g_{\mu \nu}$ is the perturbation of the 10D metric (1) with polarization in the 4D spacetime. Therefore, scaling out the warp factor, we find that the action is 


$$
S=\frac{M_{10}^{8} V_{w}}{2} \int d^{4} x \sqrt{-g_{4}}\left(1+\frac{\delta V_{w}}{V_{w}}\right) e^{-2 \delta \varphi} R\left(g_{4}\right)+\cdots,
$$

where the warped volume is

$$
V_{w} \equiv\left(2 \pi \sqrt{\alpha^{\prime}}\right)^{6} e^{6 u} \equiv \int d^{6} x \sqrt{g_{6}} e^{2 A}=\int d^{6} x \sqrt{\hat{g}} e^{-4 A}
$$

and $\hat{g}_{m n}$ is the underlying Calabi-Yau metric. Equation (A3) then defines the volume modulus $u$. Since $e^{-4 A}$ grows only as $1 / r^{4}$ as $r \rightarrow 0$ in an AdS throat (and less rapidly in other throats), we can see that $V_{w} \sim V_{\mathrm{CY}}$ is approximately the volume of the Calabi-Yau manifold. Therefore, $\sqrt{\alpha^{\prime}} e^{u}$ is approximately the linear scale of the Calabi-Yau and gives the KK mass scale for modes not localized in highly warped regions. If we henceforth refer to $u$ as the expectation value and $\delta u$ as the fluctuation, the 4D Planck constant becomes

$$
M_{P}^{2}=M_{10}^{8}\left(2 \pi \sqrt{\alpha^{\prime}}\right)^{6} e^{6 u}=\frac{e^{6 u}}{2 \pi \alpha^{\prime} g_{s}^{2}},
$$

and the graviton and scalar modes are decoupled by going to the Einstein frame $g_{\mu \nu}^{E}=e^{6 \delta u-2 \delta \varphi} g_{\mu \nu}^{4}$. Note that, since $\delta u, \delta \varphi$ are the (massive) fluctuations only, there is no rescaling of masses from the string to the Einstein frame.

Since the supergravity 3-form fluxes play an important role in stabilizing moduli and determining the geometry of warped regions of the compactification, we will also define the flux quantum numbers $n_{f, \mathrm{R}, \mathrm{NS}}$ used in the text. Both the Ramond-Ramond (R) and Neveu-Schwarz-NeveuSchwarz (NS) fluxes (respectively $F$ and $H$ ) satisfy a quantization condition when integrated on any 3-cycle $c$ :

$$
\int_{c} F=4 \pi^{2} \alpha^{\prime} n_{\mathrm{R}}^{c}, \quad \int_{c} H=4 \pi^{2} \alpha^{\prime} n_{\mathrm{NS}}^{c}, \quad n_{\mathrm{R}, \mathrm{NS}}^{c} \in \mathbf{Z} .
$$

The fluxes are completely specified by the integers $n_{\mathrm{R}, \mathrm{NS}}^{c}$. Ignoring warping, the fluxes induce masses for the complex structure moduli of the Calabi-Yau as well as the dilaton. These masses are given (up to numerical constants) by $[74,77-79]$

$$
\begin{aligned}
m^{2} & \approx g_{s}^{2}\left|F-\left(C+\frac{i}{g_{s}}\right) H\right|^{2} \\
& \approx \frac{g_{s}^{2}}{\alpha^{\prime}} e^{-6 u} \sum_{c}\left|a_{c}\left(n_{\mathrm{R}}^{c}-\left(C+\frac{i}{g_{s}}\right) n_{\mathrm{NS}}^{c}\right)\right|^{2} \\
& \equiv \frac{g_{s}^{2} n_{f}^{2}}{\alpha^{\prime}} e^{-6 u},
\end{aligned}
$$

where the sum runs over all the relevant 3 -cycles and $a_{c}$ is a numerical constant relating the components of the fluxes to the integer $n_{\mathrm{R}, \mathrm{NS}}^{c}$. Here $C$ is the scalar from the RamondRamond sector of the supergravity, which is also stabilized by the flux.
Since the fluxes also source the warp factor, they determine much of the geometry of the warped throats. There are two important points for us, which we will illustrate in the case of the deformed conifold throats of $[75,76]$. The first point is that the fluxes control the size of the tip of the throat. For example, the tip of a deformed conifold throat is $\mathbf{R}^{3} \times S^{3}$, and the radius of the sphere is given by the flux. Taking $C=0$ and the Ramond-Ramond flux to wrap the $S^{3}$, the radius of the sphere is roughly $R_{S^{3}} \sim \sqrt{g_{s} n_{\mathrm{R}}^{S^{3}} \alpha^{\prime}}$. (Henceforth, and in the rest of the paper, we drop the superscript $S^{3}$ when we discuss a particular throat. In this case, the Neveu-Schwarz-Neveu-Scharz flux wraps the cycle dual to the $S^{3}$.) The other point is that the fluxes determine the warp factor in the throat. For one, they actually fix the value of the warp factor at the bottom of the throat, although the details are unimportant to us. In addition, the warp factor behaves like the warp factor for AdS with a varying AdS radius, and the fluxes control the AdS radius. At the tip of the deformed conifold throat, for example, the AdS radius is $R_{\mathrm{AdS}} \sim \sqrt{g_{s} n_{\mathrm{R}} \alpha^{\prime}}$, making the same assumptions about the flux and $C=0$ as above. Whenever we discuss $n_{\mathrm{R}, \mathrm{NS}}$ in this paper, we are referring to this choice of flux and $C=0$ in the throat.

Finally, let us comment on the possible effect of the warp factor on the moduli masses. The effect of the warp factor should only be important for moduli with wave functions localized in a throat (or another significantly warped region), in which case we expect that we would replace $\alpha^{\prime} \rightarrow$ $\ell_{\mathrm{SM}}^{2}$ (for the SM throat, and so on) in Eq. (A6). However, there may be additional corrections due to derivatives of the warp factor. In the case of the deformation modulus of the SM throat, it is possible that the dominant term may be

$$
m^{2} \sim \frac{g_{s}^{2} n_{f}^{2}}{\ell_{\mathrm{SM}}^{2}} \frac{1}{\left(R_{\mathrm{AdS}}^{2} / \alpha^{\prime}\right)^{p}}=\frac{g_{s}^{2} n_{f}^{2}}{\ell_{\mathrm{SM}}^{2}} \frac{1}{\left(g_{s} n_{\mathrm{R}}\right)^{p}}
$$

rather than $m^{2} \sim \frac{g_{s}^{2} n_{f}^{2}}{\ell_{\mathrm{SM}}^{2}} e^{-6 u}$

for some power $p$. In the text, however, we will assume the suppression by $e^{-6 u}$ compared to the string scale, since the dimensional reduction in the presence of warping is not completely understood.

\section{APPENDIX B: ALTERNATIVE PROPOSALS FOR SM THROAT}

Here we will give a few comments about two possible alternative geometries to that discussed in Sec. III. These are modifications to the 10D geometry which might seem natural for the SM throat during inflation. We will also present arguments why these modifications are not applicable in the present context of interest. 


\section{Black-brane horizon}

The first alternative we consider is that a black-brane horizon replaces the entire lower portion of the throat. There is a simple thermal reasoning for this picture; it is well known that any observer in de Sitter (or inflationary) spacetime sees a heat bath at the Gibbons-Hawking temperature $T=H / 2 \pi$. Then we might imagine that the SM is just a gauge theory in a heat bath; string-gauge duality would then suggest representing the thermal gauge theory as a black hole with matching Hawking temperature in the SM throat [176]. In such a scenario during inflation, the entire lower portion of the SM throat (including the SM branes) are hidden by a horizon appearing in the throat. In this case, the observable physics is then cut off at some warp factor much large than the vacuum value $e^{A_{\mathrm{SM}}}-$ so in this respect it is similar to the modified geometry in Sec. III. In the black-horizon picture, the standard model degrees of freedom are no longer open strings on D branes but rather closed strings near the horizon. At the end of inflation, the horizon shrinks, the D branes appear, and the SM strings become open strings on the branes again, which should be excited up to the energy of the black hole. This process can be understood as a phase transition [66,177181]. In fact, this type of transition between thermal strings and black holes has been advocated on general grounds [182].

Black holes in these throats have been discussed in [180], in which it was shown that the geometry approximates a nonextremal black 3-brane at high temperature. The metric for the black 3-brane is $[183,184]$

$$
\begin{aligned}
d s^{2} & =e^{2 A(r)}\left[-g(r) d t^{2}+d x^{i} d x^{i}\right] \\
& +e^{-2 A(r)}\left[g^{-1}(r) d r^{2}+r^{2} d s_{5}^{2}\right],
\end{aligned}
$$

where $d s_{5}^{2}$ is some compact five-dimensional Einstein metric. For simplicity, take the six-dimensional throat to be asymptotically a conifold (up to the warping and the fact that it glues onto some compact space), so $d s_{5}^{2}$ is the metric of $T^{1,1}$. By setting the brane Hawking temperature to the de Sitter temperature, we can approximate the effective string tension for the SM throat using the warp factor $A_{h}$ at the horizon. After some algebra, we find an effective horizon string length

$$
\ell_{h}=\left(\frac{64}{g_{s} q}\right)^{1 / 4} \frac{1}{H}
$$

We should note that the D3-brane charge $q$ is an "effective" charge; the 5-form field strength varies over the conifold throat due to the presence of 3-forms [75,76]. Following the considerations of [51], the ratio of scales (warp factors) between the tip and horizon is

$$
q=N_{D}+\frac{3 g_{s} n_{R}^{2}}{2 \pi} \ln \left(\ell_{\mathrm{SM}} / \ell_{h}\right)
$$

(in agreement with [180]). We can solve the transcendental relation (B2) for $\ell_{h}$ approximately by substituting $H$ on the right-hand side and ignoring the number $N_{D}$ of SM branes. If we assume $M_{\mathrm{SM}} \sim 1 \mathrm{TeV}$ and $H \sim 10^{13} \mathrm{GeV}$, we get $g_{s} q \sim 1000$ and $\ell_{h} \sim 1 / 2 H$. It is amusing to note that we find $\ell_{h} H \lesssim 1$ so simply using thermal physics.

We can now explain a clear problem with this alternative proposal. The black horizon, because it is thermal, carries an energy density in the $\mathcal{N}=14 \mathrm{D}$ field theory which is dual to the throat geometry (as per the AdS/CFT correspondence). In fact, following [184], we can argue that the thermal energy density is

$$
\rho_{\mathrm{bb}}=\frac{3 \pi^{4} \omega_{5}}{32} q^{2} T^{4}=\frac{3 \omega_{5}}{512} q^{2} H^{4},
$$

where $\omega_{5}$ is the volume of the angular part of the compactification (which is a $T^{1,1}$ space here). Furthermore, the black-brane horizon has the equation of state of radiation, as follows from its entropy (see [182]). Now suppose we compare to the energy of the dual gauge field theory in a de Sitter background. Certainly, any observer sees radiation of temperature $T=H / 2 \pi$, but this temperature is invisible in any global sense. Specifically, in any de Sitter-invariant vacuum, the stress tensor is that of a cosmological constant $\left\langle T_{\mu \nu}\right\rangle \propto g_{\mu \nu}$. In fact, the correct dual geometries for $\mathcal{N}=1$ gauge theories on de Sitter (with nondynamical gravity) do not have thermal brane horizons, and they are reviewed in Appendix B 2 below.

Another point to note is that the energy density (B4) grows as the square of the effective D3-brane charge. This density might seem consistent with the dual picture of a gauge theory during inflation (in fact, the factor of $q^{2}$ is consistent with the number of adjoint fields); however, determining the energy density of the gauge fields is more subtle. Because the SM throat is compactified, we should think of the dual gauge theory as being coupled to gravity with a finite cutoff $M_{P}$, rather than living on a fixed background spacetime. In nondynamical gravity, we would take $M_{P} \rightarrow \infty$ and renormalize away divergent terms in the effective action. In this process, the renormalized effective action would indeed yield a stress tensor (and energy density) $\rho \propto q^{2} H^{4}$. However, since we have a finite Planck scale cutoff, no terms in the effective action diverge. Following along the calculation of [185], we find that the energy density is suppressed by powers of $H / M_{P}$ compared to (B4), so the black brane does not actually match the dual gauge theory during inflation. In other words, not only does the black brane fail to match the correct equation of state, but it also has the wrong magnitude of energy density. 


\section{AdS-like solutions}

Another alternative is also inspired by string-gauge theory duality; [186-189] have found supergravity backgrounds dual to gauge theories on some curved (but specified) spacetimes, including de Sitter spacetime. Their supergravity duals are weakly curved everywhere and have been used to study the motion of branes during inflation [190,191], so we might expect that these geometries could describe the SM throat during inflation. A central observation in these constructions is that $\mathrm{AdS}_{5}$ admits a parametrization where the radial slices have a $\mathrm{dS}_{4}$ geometry [192].

The reason we do not think that the solutions of [186189] will describe the SM throat during inflation is somewhat technical. For specificity, let us focus on the solutions in $[186,187]$, which are appropriate for the deformed conifold throats in our discussion. These solutions are found as perturbations around the de Sitter-like slicing of $\mathrm{AdS}_{5} \times$ $T^{1,1}$, and, in fact, the perturbations disappear at the bottom of the throat, leaving an $\operatorname{AdS}_{5} \times T^{1,1}$ core. Most importantly for us, the warp factor $A$ always diverges at large distances from the core. On the other hand, to glue the throat onto a compact manifold, it should be possible to find a solution with $A \rightarrow 0$ asymptotically (which is possible for the flat spacetime solutions $[75,76])$. Suppose that we tried to modify to solutions of $[186,187]$ to have $A \rightarrow 0$ asymptotically. Then asymptotically the solution would go to $\mathrm{dS}_{4}$ times a Ricci-flat manifold. However, the supergravity stress tensor (sourced by supergravity fluxes) would vanish asymptotically, leading to a contradiction. This analysis is born out by examining the equations of motion acting on a supergravity ansatz that generalizes $[186,187] .{ }^{27}$ However, it is possible that the solutions of $[186,187]$ are approximate solutions for throats in the compactification in a region far from both the tip and the bulk.

This difficulty seems tied to the fact that these supergravity solutions are dual to gauge theories on fixed spacetimes. That is, the gauge theories live in gravity backgrounds with an infinite Planck constant. The Hubble scale of inflation therefore must be imposed by hand. In fact, because these dualities are derived by perturbation around conformal theories, the Hubble scale is not an independent physical mass scale in the problem; rather, it enters as a choice of slicing for $\mathrm{AdS}_{5}$.

In closing, we should also note that, were these solutions to reflect the true SM throat geometry during inflation, the consequences for the 4D effective field theory would be disastrous. As we mentioned above, the core of these throats are $\mathrm{AdS}_{5} \times T^{1,1}$. In particular, the warp factor $A \rightarrow$ $-\infty$, leading to a horizon in the $10 \mathrm{D}$ geometry and a vanishing effective string tension.

\footnotetext{
${ }^{27}$ We thank A. Buchel for conversations about this point.
}

\section{APPENDIX C: MORE REHEATING}

Here we will discuss aspects of reheating in more general inflationary models.

\section{Many throats}

The reader might wish to consider what happens if there exist other throats with even more significant warping (in the true ground state geometry) - see related discussions in $[57,58]$. In the mechanism of [52], antibranes in these throats have small tensions, leading to a fine spacing of possible values for the cosmological constant. Although we are not concerned with the cosmological constant in this paper, we should mention the effects of extra throats on reheating.

During the reheating phase, we expect that all the long throats (that is, all the throats with a ground-state string scale less than $H_{\star}$ ) are lifted to have $\ell_{s} H_{\star} \lesssim 1$. The argument is just the same as for the SM throat. Therefore, when the inflaton reheats the inflationary throat, the KK gravitons should distribute themselves evenly among all the long throats. We expect then that the energy density in the SM throat should be reduced from our original considerations by a factor of the number of long throats. ${ }^{28}$ This effect would not seem to cause any problems with reheating, since the number of long throats is expected to be $\mathcal{O}(10)$.

Problems could arise, however, in low-energy cosmology, such as nucleosynthesis, if the energies in all the long throats remain comparable. Some mechanism is necessary to ensure that the SM throat energy density dominates by late times. An additional complication is the fact that closed string modes (such as KK gravitons) will prefer longer throats to the SM throat after about $t \sim 1 / M_{\mathrm{SM}}$, when the SM throat approaches its ground state geometry. The closed string modes will tunnel to the longer throats, just as they tunneled from the inflationary throat. Fortunately, open string modes attached to the SM branes should have a highly suppressed tunneling rate and be effectively "locked" to the SM branes. Perhaps this fact can account for the dominance of the SM throat: if the SM throat has significantly more branes than the other throats, then closed strings might preferentially attach to the SM branes. This will reduce the closed string mode energy in the SM throat compared to the other throats during an era in which all the long throats have similar warp factors. A subsequent redistribution of closed string energy among the throats would then increase the total energy in the SM throat. We leave a further investigation for future work.

We can, however, question whether throats with such small warp factors are phenomenologically viable in the present. Such significant warping would lead to very light

\footnotetext{
${ }^{28}$ If these throats are distinguished by, e.g., having different curvatures, the tunneling to the additional throats may be suppressed (or enhanced) [135].
} 
KK gravitons, so these long throats could be ruled out. On the other hand, the light KK gravitons should have highly suppressed wave functions in the SM throat, so SM modes would couple to them very weakly. It would be interesting to determine whether such long throats are ruled out or not.

\section{Reheating from other inflationary models}

We can also give a few comments about reheating for other string embeddings of inflation.

To put it shortly, both D3/D7 and non-BPS brane inflation should reheat the SM throat similarly to the usual brane inflation case. Both would generate closed strings in the inflationary region during reheating, and the resulting KK modes could tunnel to the SM throat.

Now consider the case of racetrack inflation. We recall first that racetrack inflation is caused by the slow roll of a compactification modulus, such as the total volume modulus of the compact manifold. This modulus will generally couple both to SM brane modes and bulk supergravity modes, so it can preheat the SM directly by parametric resonance along with exciting closed string states. Additionally, as in brane inflation, the bulk supergravity modes can then reheat the SM modes.

\section{APPENDIX D: MORE ON STRING THERMODYNAMICS}

In this appendix, we gather detailed formulas and calculations relevant to the discussion of string thermodynamics in Sec. VA.

\section{Open string thermodynamics}

Here we collect some other useful formulas for open string thermodynamics. We work in the context of D3 branes only for simplicity.

From (28), we can also see that the number density of open strings is

$$
n_{o}=\frac{2 a N_{D}^{2}}{b V_{\perp}} L
$$

Note that the open string system is extrinsic in the (noncompact) directions parallel to the D3 branes.

We can also calculate the entropy from the string distribution $(28)$ as in $[59,193]$. The density of states (per volume) for single open strings is

$$
\omega_{o}(\varepsilon)=\frac{2 a N_{D}^{2}}{b V_{\perp}} e^{\beta_{H} \varepsilon} .
$$

The density of states for a gas of strings of energy $E$ in a volume $V_{\|}$along the branes is therefore

$$
\Omega_{o}=\left(\frac{C}{E}\right)^{1 / 2} e^{\beta_{H} E} I_{1}(2 \sqrt{C E}) \propto \exp \left[\sqrt{\frac{8 a N_{D}^{2} V_{\|} E}{b \mu V_{\perp}}}+\beta_{H} E\right],
$$

where $4 C E$ is the combination inside the square root after the proportionality and $I_{1}$ is a modified Bessel function [60]. The proportionality is valid in the limit that $E \rightarrow \infty$ with $E / V_{\|}$constant. Therefore, the entropy is

$$
S_{o} \approx \beta_{H} E+\sqrt{\frac{8 a N_{D}^{2} V_{\|} E}{b \mu V_{\perp}}} .
$$

It is also possible to argue that quantum corrections to the entropy are no more important than the low-energy string states in the Hagedorn limit, though we do not give the details here.

\section{Closed string thermodynamics}

Here we give a very brief review of closed string thermodynamics (in contrast to open strings), which is relevant for the behavior of cosmic strings. The density of states was given in [194]; a recent review (with more references) is [61], and the relevance to cosmic strings is reviewed in [154].

In the relevant case of 3 noncompact spatial dimensions, the density of states per volume for a single closed string is

$$
\omega_{c}(\varepsilon) \approx \frac{1}{\alpha^{13} \varepsilon^{5 / 2}} e^{\beta_{H} \varepsilon} .
$$

The density of states for the entire gas of strings is

$$
\Omega_{c} \propto \frac{V}{\left(E-V / \alpha^{\prime 2}\right)^{5 / 2}} \exp \left[\beta_{H} E+\alpha^{\prime-3 / 2} V\right] .
$$

This density of states leads to a distribution of closed strings with one (or a few) very long, energetic strings, and a gas of closed string radiation with total energy density $\rho \sim \alpha^{\prime-2}$.

Additionally, it has been argued that D branes are very efficient at chopping closed strings into open strings. That is, if the D branes are packed with a density about unity in string units in the space transverse to their world volumes, open strings will dominate closed strings in equilibrium.

\section{Hagedorn strings vs black branes}

We will argue here that a Hagedorn gas of open strings will not undergo a phase transition to a black 3-brane, as is commonly supposed in the literature (and does indeed happen in some cases) (see [61,182]). For simplicity, we will consider the case of D3 branes only. We will also work in flat space as an approximation.

Let us start by considering the open string gas and black 3-brane in the microcanonical ensemble. In the open string entropy (D4), the ratio of the first to second terms is $(\hat{\beta} / 2 \pi B)\left(\rho / N_{D}^{2} \mu^{2}\right)^{1 / 2}$, where we have defined $\beta_{H}=$ $\hat{\beta} \sqrt{\alpha^{\prime}}$ and $B=8 a \alpha^{\prime} / b \mu V_{\perp}$ (both $\hat{\beta}$ and $B$ are $\mathcal{O}(1)$ ). Therefore, for densities larger than $N_{D}^{2} \mu^{2}$-where the average string is longer than the string length - the first term in (D4) dominates and the entropy is $S_{o} \approx \beta_{H} E$. On 
the other hand, the entropy of the black 3-brane is $S_{\mathrm{bb}}=$ $A \sqrt{N_{D}} V^{1 / 4} E^{3 / 4}$ [184], where $A$ is a constant of order unity. Then the ratio of entropies is

$$
\frac{S_{o}}{S_{\mathrm{bb}}} \approx \frac{\hat{\beta}}{\sqrt{2 \pi} A}\left(\frac{\rho}{N_{D}^{2} \mu^{2}}\right)^{1 / 4} \gg 1
$$

for Hagedorn densities. Heuristically, this result makes sense; starting at low densities, we expect a radiation bath to undergo a phase transition to a gas of long open strings. However, the radiation bath has the same entropy as the black brane [184], so we would not expect a further phase transition to a black-brane phase.

In the canonical ensemble, some manipulations give the free energies

$$
F_{o}=-\frac{1}{4} \frac{B^{2} N_{D}^{2} V}{\alpha^{\prime} \beta\left(\beta-\beta_{H}\right)}, \quad F_{\mathrm{bb}}=-\frac{27}{256} \frac{A^{4} N_{D}^{2} V}{\beta^{4}}
$$

for temperatures below the Hagedorn temperature $1 / \beta_{H}$. The ratio of free energies is

$$
\frac{F_{o}}{F_{\mathrm{bb}}}=\frac{64}{27} \frac{B^{2}}{\alpha^{\prime} A^{4}} \frac{\beta^{3}}{\beta-\beta_{H}}=\frac{64}{27} \frac{B \hat{\beta}^{3}}{\pi A^{4}}\left(\frac{\rho}{N_{D}^{2} \mu^{2}}\right)^{1 / 2} .
$$

For small enough $\beta$ (or high enough density), the open string free energy is larger (i.e., more negative) and so the open strings are thermodynamically favored. We should contrast this behavior to that expected from the AdS/CFT correspondence, in which a thermal state of the gauge theory corresponds to a black-brane horizon in the throat for high temperatures. In that case, the strings in the throat are exposed to a heat bath of a temperature greater than the Hagedorn temperature. Therefore, the only thermal state available to any open strings in the throat is a black brane. Furthermore, we expect that closed strings will actually have a phase transition to a black brane, so there is no issue for gravity duals with no free branes.

There are some possible loopholes in our argument, however. Most importantly, it was demonstrated in [195] that the radiation gas can undergo a first-order phase transition to a black-brane phase at a lower temperature than the Hagedorn temperature. Indeed, if the SM degrees of freedom can thermalize before a density of order $N_{D}^{2} \mu^{2}$ accumulates, we do expect this first-order transition. On the other hand, if, as we believe, thermalization occurs later, the long open string gas would form. ${ }^{29}$ Also, we have neglected any effects from the nontrivial geometry of the SM throat, instead using a flat background. In particular, the changing warp factor may favor a blackbrane horizon. If the strings filled the entire throat and entered the region of the compactification where $A \sim 0$, then they should be compared to a far-from-extremal black

\footnotetext{
${ }^{29}$ We thank O. Aharony for interesting discussions on this point.
}

brane, which has a different entropy formula. We leave it to the future to check our results.

\section{World-sheet fluctuations in warp factor}

In this appendix, we will discuss the compact volume $V_{\perp}$ accessible to long strings in the SM throat. Classically speaking, strings are confined to minima of the warp factor because the strings feel $e^{2 A}$ as a gravitational potential. However, in a quantum mechanical or thermal system, the string centers of mass can spread to larger values of the warp factor, as was first discussed in [132]. We will first review the zero temperature calculation of [132], and then we will generalize it to finite temperature. In the end, we will find that the two calculations give similar answers.

In the metric (1), the static gauge action for fluctuations of a long string in the compact dimension $Y$ is

$$
S=-\frac{1}{2 \pi \alpha^{\prime}} \int d^{2} \sigma\left[e^{2 A}+\frac{1}{2}\left(\partial_{a} Y \partial^{a} Y+\left(\partial_{Y}^{2} e^{2 A}\right) Y^{2}\right)+\cdots\right] .
$$

Here, we are using diffeomorphism invariance in the internal dimensions to simultaneously diagonalize $g_{m n}$ and $\partial_{m} \partial_{n} e^{2 A}$. The $\cdots$ represent interactions due to higher derivatives of the warp factor; note that the mass is given by $m^{2}=\partial_{Y}^{2} e^{2 A}$. The deviation of the string in direction $Y$ is just given by the propagator $\left\langle Y^{2}\right\rangle$ on the Euclidean world sheet. Then we have

$$
\left\langle Y^{2}\right\rangle=\frac{\alpha^{\prime}}{2 \pi} \int^{\Lambda} \frac{d^{2} k}{k^{2}+m^{2}}=\frac{\alpha^{\prime}}{2} \ln \left[1+\frac{\Lambda^{2}}{m^{2}}\right],
$$

where $\Lambda$ is a world-sheet UV cutoff. The natural cutoff scale for the world sheet is the string scale measured by the 4D coordinate time (which is the world-sheet time in static gauge) because the string scale is the gap for excitations of the string in the noncompact directions. When studying the conifold throats, then, [132] had $\Lambda^{2}=e^{2 A} / \alpha^{\prime}$ and $m^{2}=$ $e^{2 A} / g_{s} n_{R} \alpha^{\prime}$. Further, at the tip of the conifold throat, the topology of the transverse geometry is $\mathbf{R}^{3} \times S^{3}$, where the warp factor varies only in the $\mathbf{R}^{3}$ directions. Therefore, the strings can fill the $S^{3}$ at the tip as well as climb slightly up the warp factor potential well. To calculate the effective volume in the $\mathbf{R}^{3}$, we use $\sqrt{\left\langle Y^{2}\right\rangle}$ as the linear scale. In the end, we arrive at [132]

$$
\frac{V_{\perp}}{\left(4 \pi^{2} \alpha^{\prime}\right)^{3}} \approx \frac{\left(g_{s} n_{R}\right)^{3 / 2}}{4 \pi}\left[\frac{\ln \left(1+g_{s} n_{R}\right)}{2 \pi}\right]^{3 / 2} .
$$

For $g_{s} n_{R} \sim 10, V_{\perp} /\left(4 \pi^{2} \alpha^{\prime}\right)^{3} \sim 1 / 2$. We should regard this volume as a minimum value, regardless of whether the gas of strings can stay in thermal equilibrium.

Near the Hagedorn temperature, though, we should use a thermal expectation value rather than the quantum value. Now the Euclidean time direction is compact, and the twopoint function is 


$$
\begin{gathered}
\left\langle Y^{2}\right\rangle=\frac{\alpha^{\prime}}{\beta} \sum_{n=-\infty}^{\infty} \int d k \frac{1}{\omega_{n}^{2}+k^{2}+m^{2}}, \\
\omega_{n}=\frac{2 \pi n}{\beta}, \quad n \in \mathbf{Z} .
\end{gathered}
$$

The momentum $k$ is now only in the spatial world-sheet direction. After the (convergent) momentum integral,

$$
\left\langle Y^{2}\right\rangle=\frac{\pi \alpha^{\prime}}{\beta} \sum_{n} \frac{1}{\left(\omega_{n}^{2}+m^{2}\right)^{1 / 2}} .
$$

The sum diverges, so once again we need to impose the same cutoff $\Lambda$ for the frequency. Further, we are interested in the Hagedorn limit, so we use $\beta=\beta_{H} \sim 2 \pi \ell_{\mathrm{SM}}$. Therefore, only the $n=0$ term contributes, due to the cutoff, and using the same mass as before, we get

$$
\left\langle Y^{2}\right\rangle \approx \frac{\alpha^{\prime}}{2} \sqrt{g_{s}} n_{R} .
$$

[1] C. L. Bennett et al., Astrophys. J. 583, 1 (2003).

[2] C. L. Bennett et al., Astrophys. J. Suppl. Ser. 148, 1 (2003).

[3] E. Komatsu et al., Astrophys. J. Suppl. Ser. 148, 119 (2003).

[4] H. V. Peiris et al., Astrophys. J. Suppl. Ser. 148, 213 (2003).

[5] C. P. Burgess et al., J. High Energy Phys. 07 (2001) 047.

[6] A. Mazumdar, S. Panda, and A. Perez-Lorenzana, Nucl. Phys. B614, 101 (2001).

[7] C. P. Burgess, P. Martineau, F. Quevedo, G. Rajesh, and R. J. Zhang, J. High Energy Phys. 03 (2002) 052.

[8] J. Garcia-Bellido, R. Rabadan, and F. Zamora, J. High Energy Phys. 01 (2002) 036.

[9] C. Herdeiro, S. Hirano, and R. Kallosh, J. High Energy Phys. 12 (2001) 027.

[10] N. Jones, H. Stoica, and S. H. H. Tye, J. High Energy Phys. 07 (2002) 051

[11] S. Kachru et al., J. Cosmol. Astropart. Phys. 10 (2003) 013.

[12] H. Firouzjahi and S.H.H. Tye, Phys. Lett. B 584, 147 (2004).

[13] C. P. Burgess, J. M. Cline, H. Stoica, and F. Quevedo, J. High Energy Phys. 09 (2004) 033.

[14] J. P. Hsu and R. Kallosh, J. High Energy Phys. 04 (2004) 042.

[15] K. Dasgupta, J. P. Hsu, R. Kallosh, A. Linde, and M. Zagermann, J. High Energy Phys. 08 (2004) 030.

[16] J. J. Blanco-Pillado et al., J. High Energy Phys. 11 (2004) 063.

[17] K. Becker, M. Becker, and A. Krause, Nucl. Phys. B715, 349 (2005).

[18] D. Cremades, F. Quevedo, and A. Sinha, J. High Energy Phys. 10 (2005) 106.

[19] A. Linde, J. Phys. Conf. Ser. 24, 151 (2005).
The volume is then

$$
\frac{V_{\perp}}{\left(4 \pi^{2} \alpha^{\prime}\right)^{3}} \approx \frac{\left(g_{s} n_{R}\right)^{3 / 2}}{4 \pi}\left[\frac{\sqrt{g_{s}} n_{R}}{2 \pi}\right]^{3 / 2}
$$

in a thermal calculation. Plugging in the numerical values from above, we see $V_{\perp} /\left(4 \pi^{2} \alpha^{\prime}\right)^{3} \sim 1$. Since there is not much difference between the quantum and thermal values, we can just approximate $V_{\perp} /\left(4 \pi^{2} \alpha^{\prime}\right)^{3} \sim 1$ whenever we need to make an estimate.

We conclude with one additional note. Above, we implicitly treated the string as being long by integrating over spatial world-sheet momenta. For short strings (those near $\ell_{\mathrm{SM}}$ in length), we should replace the spatial momentum integral by a sum.
[20] S. Dimopoulos, S. Kachru, J. McGreevy, and J. G. Wacker, hep-th/0507205.

[21] J. M. Cline and H. Stoica, hep-th/0508029 [Phys. Rev. D (to be published)].

[22] N. Kaloper, M. Kleban, A. E. Lawrence, and S. Shenker, Phys. Rev. D 66, 123510 (2002).

[23] C. P. Burgess, J. M. Cline, and R. Holman, J. Cosmol. Astropart. Phys. 10 (2003) 004.

[24] K. Schalm, G. Shiu, and J. P. van der Schaar, J. High Energy Phys. 04 (2004) 076.

[25] M. Porrati, Phys. Lett. B 596, 306 (2004).

[26] B. R. Greene, K. Schalm, G. Shiu, and J. P. van der Schaar, J. Cosmol. Astropart. Phys. 02 (2005) 001.

[27] R. Easther, W. H. Kinney, and H. Peiris, J. Cosmol. Astropart. Phys. 05 (2005) 009.

[28] H. Collins and R. Holman, Phys. Rev. D 71, 085009 (2005).

[29] R. Easther, W. H. Kinney, and H. Peiris, J. Cosmol. Astropart. Phys. 08 (2005) 001.

[30] H. Collins and R. Holman, hep-th/0507081.

[31] J. Martin and R.H. Brandenberger, Phys. Rev. D 63, 123501 (2001).

[32] R. H. Brandenberger and J. Martin, Mod. Phys. Lett. A 16, 999 (2001).

[33] J. Martin and R. H. Brandenberger, astro-ph/0012031.

[34] U.H. Danielsson, Phys. Rev. D 66, 023511 (2002).

[35] U. H. Danielsson, J. High Energy Phys. 07 (2002) 040.

[36] R. H. Brandenberger and J. Martin, Int. J. Mod. Phys. A 17, 3663 (2002).

[37] J. Martin and R. Brandenberger, Phys. Rev. D 68, 063513 (2003).

[38] R.H. Brandenberger and J. Martin, Phys. Rev. D 71, 023504 (2005).

[39] J. C. Niemeyer, Phys. Rev. D 63, 123502 (2001).

[40] J. C. Niemeyer and R. Parentani, Phys. Rev. D 64, 101301 
(2001).

[41] A. Kempf and J.C. Niemeyer, Phys. Rev. D 64, 103501 (2001).

[42] J. C. Niemeyer, R. Parentani, and D. Campo, Phys. Rev. D 66, 083510 (2002).

[43] R. Easther, B. R. Greene, W. H. Kinney, and G. Shiu, Phys. Rev. D 64, 103502 (2001).

[44] R. Easther, B. R. Greene, W. H. Kinney, and G. Shiu, Phys. Rev. D 67, 063508 (2003).

[45] R. Easther, B. R. Greene, W. H. Kinney, and G. Shiu, Phys. Rev. D 66, 023518 (2002).

[46] U.H. Danielsson, Phys. Rev. D 71, 023516 (2005).

[47] C. P. Burgess, J. M. Cline, F. Lemieux, and R. Holman, J. High Energy Phys. 02 (2003) 048.

[48] E. Silverstein and D. Tong, Phys. Rev. D 70, 103505 (2004).

[49] M. Alishahiha, E. Silverstein, and D. Tong, Phys. Rev. D 70, 123505 (2004)

[50] L. Randall and R. Sundrum, Phys. Rev. Lett. 83, 3370 (1999).

[51] S. B. Giddings, S. Kachru, and J. Polchinski, Phys. Rev. D 66, 106006 (2002)

[52] S. Kachru, R. Kallosh, A. Linde, and S. P. Trivedi, Phys. Rev. D 68, 046005 (2003).

[53] G. R. Dvali, hep-ph/9503259.

[54] M. Dine, L. Randall, and S. Thomas, Phys. Rev. Lett. 75, 398 (1995)

[55] M. Dine, L. Randall, and S. Thomas, Nucl. Phys. B458, 291 (1996)

[56] N. Barnaby, C.P. Burgess, and J.M. Cline, J. Cosmol. Astropart. Phys. 04 (2005) 007.

[57] L. Kofman and P. Yi, Phys. Rev. D 72, 106001 (2005).

[58] D. Chialva, G. Shiu, and B. Underwood, hep-th/0508229.

[59] S.-M. Lee and L. Thorlacius, Phys. Lett. B 413, 303 (1997).

[60] S. A. Abel, J.L. F. Barbón, I. I. Kogan, and E. Rabinovici, J. High Energy Phys. 04 (1999) 015.

[61] J.L.F. Barbón and E. Rabinovici, hep-th/0407236.

[62] V. Balasubramanian and I. R. Klebanov, Mod. Phys. Lett. A 11, 2271 (1996).

[63] A. R. Frey, M. Lippert, and B. Williams, Phys. Rev. D 68, 046008 (2003).

[64] L. Randall and R. Sundrum, Phys. Rev. Lett. 83, 4690 (1999).

[65] J. Lykken, R. C. Myers, and J. Wang, J. High Energy Phys. 09 (2000) 009.

[66] L. A. Pando Zayas and D. Vaman, Phys. Rev. Lett. 91, 111602 (2003).

[67] S. Cullen, M. Perelstein, and M.E. Peskin, Phys. Rev. D 62, 055012 (2000).

[68] K. Dasgupta, G. Rajesh, and S. Sethi, J. High Energy Phys. 08 (1999) 023.

[69] B. R. Greene, K. Schalm, and G. Shiu, Nucl. Phys. B584, 480 (2000).

[70] A. R. Frey, hep-th/0308156.

[71] F. Denef, M. R. Douglas, and B. Florea, J. High Energy Phys. 06 (2004) 034.

[72] K. Becker, M. Becker, M. Haack, and J. Louis, J. High Energy Phys. 06 (2002) 060.

[73] V. Balasubramanian and P. Berglund, J. High Energy Phys. 11 (2004) 085 .
[74] S. B. Giddings and A. Maharana, hep-th/0507158.

[75] I. R. Klebanov and A. A. Tseytlin, Nucl. Phys. B578, 123 (2000).

[76] I. R. Klebanov and M. J. Strassler, J. High Energy Phys. 08 (2000) 052.

[77] S. P. de Alwis, Phys. Rev. D 68, 126001 (2003).

[78] A. Buchel, Phys. Rev. D 69, 106004 (2004).

[79] S. P. de Alwis, Phys. Lett. B 603, 230 (2004).

[80] J. F. G. Cascales, M.P. Garcia del Moral, F. Quevedo, and A. M. Uranga, J. High Energy Phys. 02 (2004) 031.

[81] S. Franco, Y.-H. He, C. Herzog, and J. Walcher, Phys. Rev. D 70, 046006 (2004).

[82] C.P. Herzog, Q.J. Ejaz, and I.R. Klebanov, J. High Energy Phys. 02 (2005) 009.

[83] S. Franco, A. Hanany, and A. M. Uranga, J. High Energy Phys. 09 (2005) 028.

[84] D. Berenstein, C. P. Herzog, P. Ouyang, and S. Pinansky, J. High Energy Phys. 09 (2005) 084.

[85] S. Franco, A. Hanany, F. Saad, and A. M. Uranga, hep-th/ 0505040.

[86] M. Bertolini, F. Bigazzi, and A. L. Cotrone, Phys. Rev. D 72, 061902 (2005).

[87] S. Kachru, J. Pearson, and H. Verlinde, J. High Energy Phys. 06 (2002) 021.

[88] A. M. Uranga, Classical Quantum Gravity 20, S373 (2003).

[89] D. Lust, Classical Quantum Gravity 21, S1399 (2004).

[90] R. Blumenhagen, Fortschr. Phys. 53, 426 (2005).

[91] C. P. Burgess, P. Martineau, F. Quevedo, and R. Rabadan, J. High Energy Phys. 06 (2003) 037.

[92] M. Berg, M. Haack, and B. Kors, Phys. Rev. D 71, 026005 (2005).

[93] X.-G. Chen, Phys. Rev. D 71, 063506 (2005).

[94] M. Berg, M. Haack, and B. Kors, hep-th/0409282.

[95] A. R. Liddle, A. Mazumdar, and F. E. Schunck, Phys. Rev. D 58, 061301 (1998).

[96] E. J. Copeland, A. Mazumdar, and N. J. Nunes, Phys. Rev. D 60, 083506 (1999).

[97] A. Jokinen and A. Mazumdar, Phys. Lett. B 597, 222 (2004).

[98] K. Enqvist, A. Jokinen, A. Mazumdar, T. Multamaki, and A. Vaihkonen, J. High Energy Phys. 08 (2005) 084.

[99] Q.-G. Huang and K. Ke, hep-th/0504137.

[100] J.M. Cline, H. Firouzjahi, and P. Martineau, J. High Energy Phys. 11 (2002) 041.

[101] S. Sarangi and S. H. H. Tye, Phys. Lett. B 536, 185 (2002).

[102] S. Sarangi and S. H. H. Tye, Phys. Lett. B 573, 181 (2003).

[103] K. Dasgupta, C. Herdeiro, S. Hirano, and R. Kallosh, Phys. Rev. D 65, 126002 (2002).

[104] J.P. Hsu, R. Kallosh, and S. Prokushkin, J. Cosmol. Astropart. Phys. 12 (2003) 009.

[105] J. Lesgourgues, S. Pastor, M. Peloso, and L. Sorbo, Phys. Lett. B 489, 411 (2000).

[106] S. Kanno and J. Soda, Phys. Rev. D 71, 044031 (2005).

[107] A. Mazumdar, Phys. Rev. D 64, 027304 (2001).

[108] A. Mazumdar, Nucl. Phys. B597, 561 (2001).

[109] S. S. Gubser, Phys. Rev. D 69, 123507 (2004).

[110] S. S. Gubser, hep-th/0312321.

[111] J. J. Friess, S. S. Gubser, and I. Mitra, Nucl. Phys. B689, 243 (2004).

[112] N. Turok, Phys. Rev. Lett. 60, 549 (1988). 
[113] N. Sanchez and G. Veneziano, Nucl. Phys. B333, 253 (1990).

[114] M. Gasperini, N. Sanchez, and G. Veneziano, Int. J. Mod. Phys. A 6, 3853 (1991).

[115] M. Gasperini, N. Sanchez, and G. Veneziano, Nucl. Phys. B364, 365 (1991).

[116] M. Dine, W. Fischler, and D. Nemeschansky, Phys. Lett. B 136, 169 (1984).

[117] O. Bertolami and G. G. Ross, Phys. Lett. B 183, 163 (1987).

[118] E. J. Copeland, A. R. Liddle, D. H. Lyth, E. D. Stewart, and D. Wands, Phys. Rev. D 49, 6410 (1994).

[119] K. Enqvist and A. Mazumdar, Phys. Rep. 380, 99 (2003).

[120] M. K. Gaillard, H. Murayama, and K. A. Olive, Phys. Lett. B 355, 71 (1995).

[121] A. Vilenkin, Nucl. Phys. B226, 527 (1983).

[122] A. Vilenkin and L. H. Ford, Phys. Rev. D 26, 1231 (1982).

[123] A. D. Linde, Phys. Lett. B 116, 335 (1982).

[124] K. Enqvist, S. Kasuya, and A. Mazumdar, Phys. Rev. Lett. 90, 091302 (2003).

[125] K. Enqvist, A. Jokinen, S. Kasuya, and A. Mazumdar, Phys. Rev. D 68, 103507 (2003).

[126] A. Mazumdar and A. Perez-Lorenzana, Phys. Rev. Lett. 92, 251301 (2004).

[127] K. Enqvist, S. Kasuya, and A. Mazumdar, Phys. Rev. Lett. 93, 061301 (2004).

[128] S. Dimopoulos, S. Kachru, N. Kaloper, A. E. Lawrence, and E. Silverstein, Phys. Rev. D 64, 121702 (2001).

[129] S. Dimopoulos, S. Kachru, N. Kaloper, A. E. Lawrence, and E. Silverstein, Int. J. Mod. Phys. A 19, 2657 (2004).

[130] N. Barnaby, A. Berndsen, J. M. Cline, and H. Stoica, J. High Energy Phys. 06 (2005) 075.

[131] E. J. Copeland, R. C. Myers, and J. Polchinski, J. High Energy Phys. 06 (2004) 013.

[132] M. G. Jackson, N. T. Jones, and J. Polchinski, J. High Energy Phys. 10 (2005) 013.

[133] N. Lambert, H. Liu, and J. Maldacena, hep-th/0303139.

[134] A. Sen, Int. J. Mod. Phys. A 20, 5513 (2005).

[135] P. Langfelder and R. C. Myers(work in progress).

[136] F. K. Diakonos, E. N. Saridakis, and N. Tetradis, Phys. Lett. B 605, 1 (2005).

[137] S. S. Seahra, Phys. Rev. D 72, 066002 (2005).

[138] J. H. Traschen and R. H. Brandenberger, Phys. Rev. D 42, 2491 (1990).

[139] Y. Shtanov, J. H. Traschen, and R. H. Brandenberger, Phys. Rev. D 51, 5438 (1995).

[140] L. Kofman, A. D. Linde, and A. A. Starobinsky, Phys. Rev. Lett. 73, 3195 (1994).

[141] L. Kofman, A. D. Linde, and A. A. Starobinsky, Phys. Rev. D 56, 3258 (1997).

[142] R. Allahverdi and A. Mazumdar, hep-ph/0505050.

[143] P. Jaikumar and A. Mazumdar, Nucl. Phys. B683, 264 (2004).

[144] I. Affleck and M. Dine, Nucl. Phys. B249, 361 (1985).

[145] D. H. Lyth and D. Wands, Phys. Lett. B 524, 5 (2002).

[146] K. Enqvist, A. Mazumdar, and A. Perez-Lorenzana, Phys. Rev. D 70, 103508 (2004).

[147] K. Enqvist, A. Jokinen, A. Mazumdar, T. Multamaki, and A. Vaihkonen, Phys. Rev. Lett. 94, 161301 (2005).

[148] R. Allahverdi, A. Jokinen, and A. Mazumdar, Phys. Rev. D 71, 043505 (2005).
[149] R. Allahverdi, S. Hannestad, A. Jokinen, A. Mazumdar, and S. Pascoli, hep-ph/0504102.

[150] S. Abel, K. Freese, and I. I. Kogan, Phys. Lett. B 561, 1 (2003).

[151] S. Abel, K. Freese, and I. Kogan, hep-th/0303046.

[152] M. A. Cobas, M. A. R. Osorio, and M. Suárez, hep-th/ 0411265.

[153] J. Polchinski, Phys. Lett. B 209, 252 (1988).

[154] A. Vilenkin and E.P.S. Shellard, Cosmic Strings and Other Topological Defects (Cambridge University Press, Cambridge, 1994), p. 517.

[155] D. Mitchell, N. Turok, R. Wilkinson, and P. Jetzer, Nucl. Phys. B315, 1 (1989).

[156] J. Dai and J. Polchinski, Phys. Lett. B 220, 387 (1989).

[157] H. Okada and A. Tsuchiya, Phys. Lett. B 232, 91 (1989).

[158] D. Mitchell, B. Sundborg, and N. Turok, Nucl. Phys. B335, 621 (1990).

[159] J. M. Cline, M. Joyce, and K. Kainulainen, J. High Energy Phys. 07 (2000) 018.

[160] M. Carena and H. E. Haber, Prog. Part. Nucl. Phys. 50, 63 (2003).

[161] J. Preskill, M. B. Wise, and F. Wilczek, Phys. Lett. 120B, 127 (1983).

[162] L. F. Abbott and P. Sikivie, Phys. Lett. 120B, 133 (1983).

[163] M. Dine and W. Fischler, Phys. Lett. 120B, 137 (1983).

[164] M. T. Grisaru and D. Zanon, Phys. Lett. B 177, 347 (1986).

[165] R. C. Myers, Nucl. Phys. B289, 701 (1987).

[166] J.-C. Hwang and H. Noh, Phys. Rev. D 71, 063536 (2005).

[167] X.-G. Chen, hep-th/0501184.

[168] S. Hannestad and L. Mersini-Houghton, Phys. Rev. D 71, 123504 (2005).

[169] P. Binetruy, C. Deffayet, and D. Langlois, Nucl. Phys. B565, 269 (2000).

[170] C. Csaki, M. Graesser, L. Randall, and J. Terning, Phys. Rev. D 62, 045015 (2000).

[171] E. W. Kolb and M.S. Turner, The Early Universe, Frontiers in Physics (Addison-Wesley, Redwood City, USA, 1990), p. 547.

[172] B. J. Carr and S. W. Hawking, Mon. Not. R. Astron. Soc. 168, 399 (1974).

[173] A. R. Liddle and A. M. Green, Phys. Rep. 307, 125 (1998).

[174] F. Halzen, E. Zas, J. H. MacGibbon, and T. C. Weekes, Nature (London) 353, 807 (1991).

[175] A. Nayeri, R. H. Brandenberger, and C. Vafa, hep-th/ 0511140.

[176] E. Witten, Adv. Theor. Math. Phys. 2, 505 (1998).

[177] A. Buchel, Nucl. Phys. B600, 219 (2001).

[178] A. Buchel, C. P. Herzog, I. R. Klebanov, L. A. Pando Zayas, and A.A. Tseytlin, J. High Energy Phys. 04 (2001) 033.

[179] A. Buchel and A. R. Frey, Phys. Rev. D 64, 064007 (2001).

[180] S. S. Gubser, C. P. Herzog, I. R. Klebanov, and A. A. Tseytlin, J. High Energy Phys. 05 (2001) 028.

[181] O. Aharony, J. Marsano, S. Minwalla, K. Papadodimas, and M. Van Raamsdonk, hep-th/0310285.

[182] G. T. Horowitz and J. Polchinski, Phys. Rev. D 55, 6189 (1997).

[183] G. T. Horowitz and A. Strominger, Nucl. Phys. B360, 197 (1991).

[184] S. S. Gubser, I. R. Klebanov, and A. W. Peet, Phys. Rev. D 54, 3915 (1996). 
[185] S. M. Christensen, Phys. Rev. D 17, 946 (1978).

[186] A. Buchel and A. A. Tseytlin, Phys. Rev. D 65, 085019 (2002).

[187] A. Buchel, Phys. Rev. D 65, 125015 (2002).

[188] A. Buchel, P. Langfelder, and J. Walcher, Phys. Rev. D 67, 024011 (2003).

[189] A. Buchel, Phys. Lett. B 570, 89 (2003).

[190] A. Buchel and R. Roiban, Phys. Lett. B 590, 284 (2004).

[191] A. Buchel and A. Ghodsi, Phys. Rev. D 70, 126008 (2004).
[192] R. Emparan, C. V. Johnson, and R. C. Myers, Phys. Rev. D 60, 104001 (1999).

[193] D. A. Lowe and L. Thorlacius, Phys. Rev. D 51, 665 (1995).

[194] N. Deo, S. Jain, and C.-I. Tan, Phys. Rev. D 40, 2626 (1989).

[195] O. Aharony, S. Minwalla, and T. Wiseman, hep-th/ 0507219. 